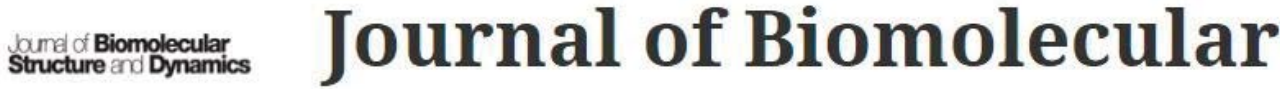 Structure and Dynamics
}

(2) Publish open access in this journal

Publishes international research on biological structure and dynamics, including atomic structural biology, bioinformatics, genomics and biological networks. 

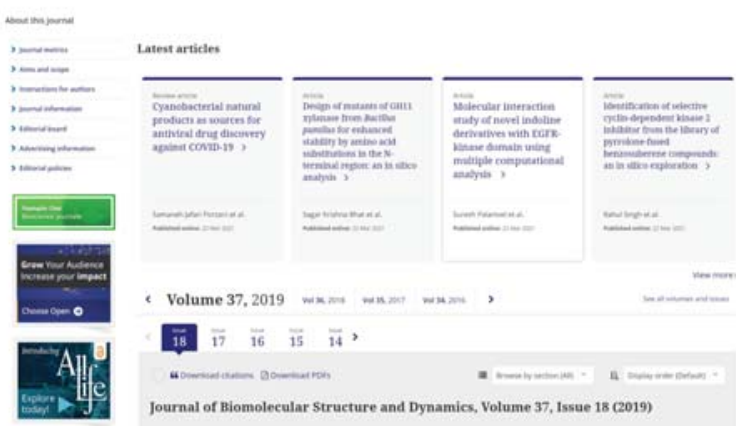

[175] $\overline{17} \quad \overline{15} \quad \overline{14}$,

Journal of Biomolecular Structure and Dynamico, Volume 37, Issue 18 c2019) -

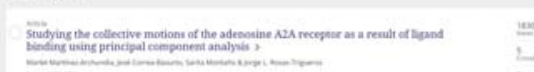

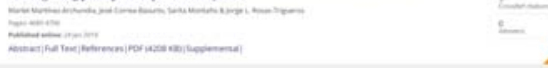

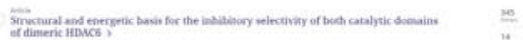

$=-1$.

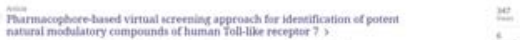

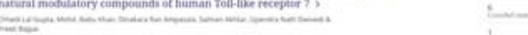

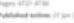

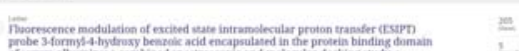

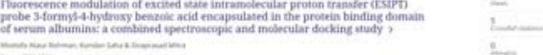

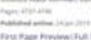

-

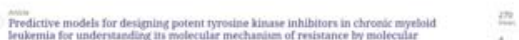

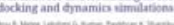

$=$ =-

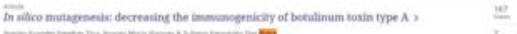

E-

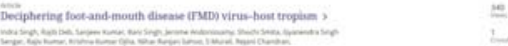

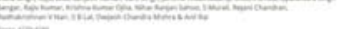

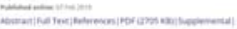

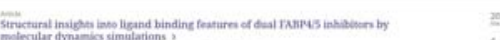

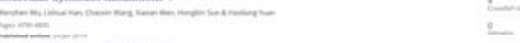

tersoutate

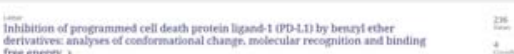
-

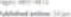

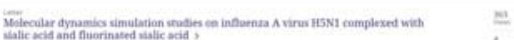

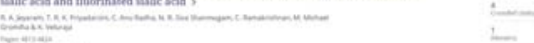

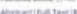

mentom

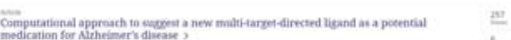

core-

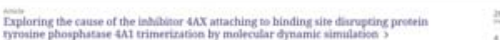

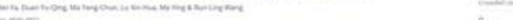

-

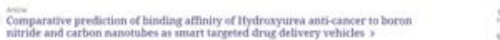

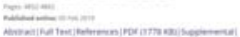

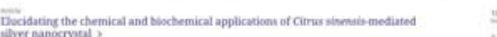

$=$

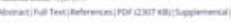

tomenom tom

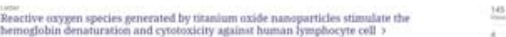

$=$

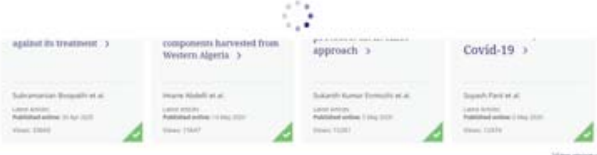




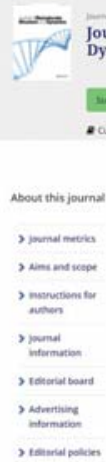

ournal of Biomolecular Structure and
Dynamics)

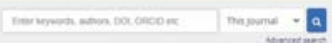

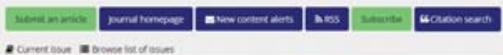

Editorial board

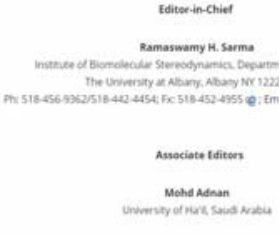

imetyas hasan

$$
\text { Centive to }
$$

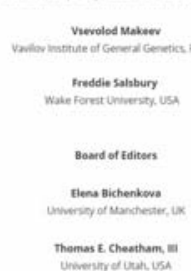

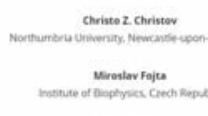

Yaskov Kxaby levy

Lus a varky

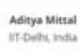

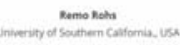

$$
\begin{aligned}
& \text { Wolltram Seenser. } \\
& \text { Irise M. Musw } \\
& \text { Dippore shomkes } \\
& \text { a nomer }
\end{aligned}
$$

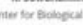

$$
\begin{aligned}
& \text { ini sponer }
\end{aligned}
$$

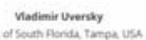

$$
\begin{aligned}
& \text { sterens s. wimmengen }
\end{aligned}
$$

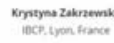

$$
\begin{aligned}
& \text { Dmity o. Zheritew }
\end{aligned}
$$

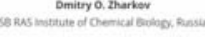

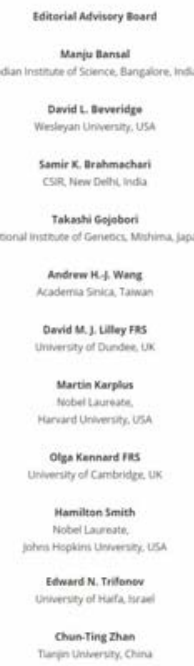

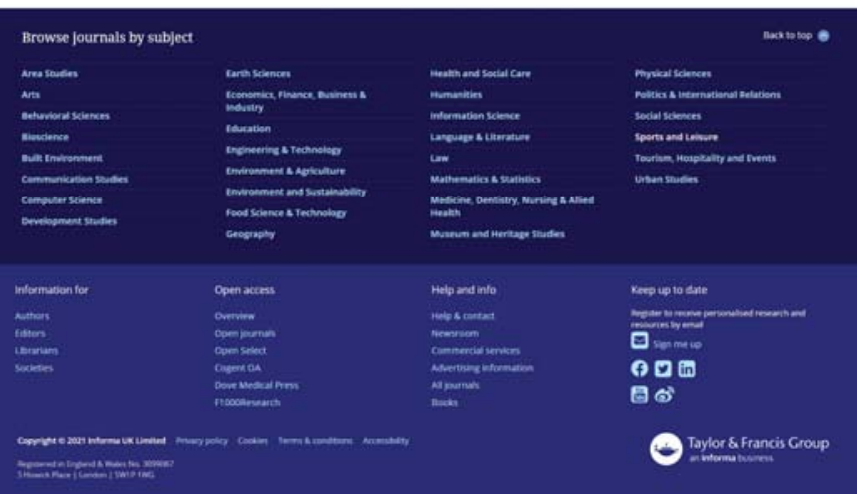




\section{Journal of Biomolecular Structure and Dynamics}

\author{
COUNTRY \\ United Kingdom \\ Universities and research \\ institutions in United Kingdom
}

PUBLICATION TYPE

Journals

\section{SUBJECT AREA AND CATEGORY}

Biochemistry, Genetics and Molecular Biology Molecular Biology

Structural Biology

Medicine

Medicine (miscellaneous)

\section{PUBLISHER}

Taylor and Francis Ltd.

More value for peer rel

Why join Peer Reviewers

Why Join Peer Reviewers - Free Res

- ReviewerCredits

reviewercredits,con

OPEN
H-INDEX
ISSN

07391102,15380254
COVERAGE

$1981,1983-2020$
INFORMATION

Homepage
Call For Papers Elsevier C

Peer Reviewed Indexed Journal

Collaboration proposals are invited to $f$

turcomatorg

OPEN

The Journal of Biomolecular Structure and Dynamics welcomes manuscripts on biological structure, dynamics, interactions and expression. The Journal is one of the leading publications in high end computational science, atomic structural biology, bioinformatics, virtual drug design, genomics and biological networks. The Journal publishes original articles and timely reviews. If an author wishes to write a review, please obtain clearance from any one of the Editors. 


\section{Journal of Biomolecular Structure and Dynamics}

\section{In silico mutagenesis: decreasing the immunogenicity of botulinum toxin type $A$}

\section{Stanley Evander Emeltan Tjoa, Yoanes Maria Vianney \& Sulistyo Emantoko Dwi Putra}

To cite this article: Stanley Evander Emeltan Tjoa, Yoanes Maria Vianney \& Sulistyo Emantoko Dwi Putra (2019) In silico mutagenesis: decreasing the immunogenicity of botulinum toxin type A, Journal of Biomolecular Structure and Dynamics, 37:18, 4767-4778, DOI: 10.1080/07391102.2018.1559100

To link to this article: https://doi.org/10.1080/07391102.2018.1559100

View supplementary material $₫$

\section{Accepted author version posted online: 17 Dec 2018. \\ Published online: 16 Jan 2019. \\ Submit your article to this journal $\sqsubset$}

\section{|ll Article views: 97}

\section{Q View related articles $匚$}




\title{
In silico mutagenesis: decreasing the immunogenicity of botulinum toxin type A
}

\author{
Stanley Evander Emeltan Tjoa*, Yoanes Maria Vianney* and Sulistyo Emantoko Dwi Putra \\ Faculty of Biotechnology, University of Surabaya, Surabaya, East Java, Indonesia \\ Communicated by Ramaswamy H. Sarma
}

\begin{abstract}
Botulinum toxin serotype $A$ is a prominent therapeutic enzyme, for both clinical and cosmetic uses. Since this protein is produced by bacteria, it exhibits an allergenic effect when subjected to human therapy. Protein mutagenesis is one method to improve the characteristics of protein. However, in silico study is needed to give suggestion of which amino acid should be mutated. Hence, a lot of money and time can be saved. This study initially screened which residue of the Botulinum toxin serotype $A$ is B-cell epitopes both linearly and conformationally. By overlapping the B-cell epitopes with the excluded conserve sequence, seven residues were allowed to be mutated. There were two proposed muteins showing a reduction in the antigenicity probability: $\triangle E 147, \mathrm{E} 510 \mathrm{~F}, \mathrm{~T} 1062 \mathrm{~F}, \triangle E 1080, \mathrm{~N} 1089 \mathrm{M}$ and $\Delta Q 1090$; and $\Delta E 147, \mathrm{E} 510 \mathrm{~F}, \mathrm{~T} 1062 \mathrm{~F}, \mathrm{E} 1080 \mathrm{~W}, \mathrm{~N} 1089 \mathrm{M}$ and $\triangle Q 1090$. Molecular dynamics simulation of the 3D proposed muteins indicated an increase of flexibility in both muteins compared to that in the native protein. Both muteins have lower antigenicity. In addition, they are similar in structure, stability and functionality compared to the native protein.
\end{abstract}

Abbreviations: 3D: three-dimensional; BoNT: botulinum neurotoxins; FNN: feed-forward neural network; GRAVY: grand average of hydropathy; MSMS: Michel Sanner's molecular surface; MD simulation: molecular dynamics simulation; NPT: constant number, pressure and temperature; NVT: constant number, volume and temperature; SASA: solvent-accessible surface area; $\mathrm{R}_{\mathrm{g}}$ : radius of gyration; RMSD: rootmean-square deviation; RMSF: root-mean-square fluctuation; SVM: support vector machine; US FDA: United States Food and Drug Administration

\section{ARTICLE HISTORY}

Received 7 August 2018

Accepted 9 December 2018

\section{KEYWORDS}

Bioinformatics; botulinum toxin type A; B-cell epitopes prediction; immunogenicity; in silico mutagenesis

\section{Introduction}

Botulinum neurotoxins (BoNT) are potent toxins which are produced mainly by Clostridium botulinum, and the other producers are $C$. argentinense, $C$. baratii and $C$. butyricum. Eight serotypes of BoNT $(\mathrm{A}-\mathrm{H})$ have been characterized. These toxins are zinc metalloprotease that consists of heavy chain, light chain and translocation domain (Hill, Xie, Foley, \& Smith, 2015). Botulinum toxin was first known to cause food poisoning resulting in muscle paralysis. Botulinum toxin affected muscle neuron after its internalization of the botox to the cytosol by the heavy chain via SV2 receptor protein; the light chain of toxin cut the SNAP-25 (by BoNT/A,/E, and/ C), VAMP/synaptobrevin (by BoNT/B,/D,/F,/G) and syntaxin (BoNT/C). These proteins are well known as SNARE complex components which act as a docking protein for neurotransmitter exocytosis (Carruthers \& Carruthers, 2005; Luvisetto, Gazerani, Cianchetti, \& Pavone, 2015).

Among those toxin serotypes, BoNT/A is the most utilized protein as a therapeutic agent for muscle relaxation, since botox is the one to be well characterized, tested and approved by the US Food and Drug Administration (FDA)
(Brooks, 1954; Frevert, 2015; Scott, 1981). Since then, BoNT/A has been widely used for many cosmetics and physiologic disorders, such as blepharospasm, hemifacial spasm, dystonia, hyperhidrosis, migraine and neuropathic pain (neuralgia) (Babiloni, Kapos, \& Nixdorf, 2016; Bentivoglio, Del Grande, Petracca, Lalongo, \& Ricciardi, 2015; Carruthers \& Carruthers, 2005; Castelao et al., 2017). BoNT/A as a therapeutic protein is also stimulated by biotechnology revolution. This encourages the market for more innovation and its unique properties dedicated to increase the performance of the protein by many industries around the world (Dressler, 2016).

Since BoNT/A is produced originally by bacteria, this protein is considered as a foreign antigen by human immune system. Antibody secretion by B-cell can neutralize the protein and may reduce its efficacy (Schellekens, 2002). Although first doubtfully reported to induce allergic and even anaphylaxis reaction due to the possibility of other foreign substances in the preparation (LeWitt \& Trosch, 1997; Li, Goldberger, \& Hopkins, 2005), BoNT/A is proven to be able to induce immunogenic and even anaphylaxis reaction, which is life-threatening (Careta, Delgado, \& Patriota, 2015;

CONTACT Sulistyo Emantoko Dwi Putra emantoko@staff.ubaya.ac.id E Faculty of Biotechnology, University of Surabaya, Kalirungkut Road, 60292 Surabaya, East Java, Indonesia.

*Both authors contributed equally.

(1) Supplemental data for this article can be accessed here.

(C) 2018 Informa UK Limited, trading as Taylor \& Francis Group 
Moon, Chang, \& Kim, 2017; Rosenfield, Kardassakis, Tsia, \& Stayner, 2014). Indeed, BoNT/A can induce the production of antibody (lgG isotype) (Dressler \& Bigalke, 2017). With the currently vast and diverse market of BoNT/A, it is beneficial to tackle the allergenic problems as one of the different technologies proposed for its development.

Bioinformatics offer many algorithms to predict amino acid residues that induce immunogenic reaction. B-cells can detect both linear (continuous) and conformational (discontinuous) epitopes unique to human protein although most of the B-cell epitopes are conformational or fold-dependent (Kringelum, Lundegaard, Lund, \& Nielsen, 2012; Yao, Zhang, Liang, \& Zhang, 2012). Modifications of these residues can alter the antigenicity (Zarei et al., 2018). Compared to time-consuming and costly experimental approaches, a wide variety of computational tools, which are publicly available, are highly recommended to predict the B-cell epitopes to reduce or minimize cost (Kolaskar \& Tongaonkar, 1990; Kringelum et al., 2012; Potocnakova, Bhide, \& Pulzova, 2016; Saha \& Raghava, 2006; Singh, Ansari, \& Raghava, 2013; Yao et al., 2012).

Few studies to mutate BoNT/A to enhance the botox performance have been attempted (Fonfria, Elliott, Beard, Chaddock, \& Krupp, 2018). This paper focuses on the strategy of BoNT/A rational design to reduce the immunogenicity of the protein by mutating the predicted B-cell epitopes. B-cell epitopes were predicted both conformationally and linearly; these epitopes overlapped with the conserved region predicted by the thermodynamic analysis and the essential site of the toxin for the activity (Rigoni, Caccin, Johnson, Montecucco, \& Rossetto, 2001). Then, the new protein was also modelled and evaluated for its physical properties and stability.

\section{Materials and methods}

In general, the steps used in this methodology were (1) determination of $\mathrm{B}$-cell linear and conformational epitopes from BoNT/A, (2) determination of conserved region and (3) mutagenesis of BoNT/A and evaluation of antigenic, physical properties and stability.

\subsection{Sequence retrieval}

The protein used in this paper was botulinum toxin serotype A (BoNT/A) (PDB ID: 3BTA) (Lacy, Tepp, Cohen, DasGupta, \& Stevens, 1998). Protein domain and features were obtained from UniProt (https://www.uniprot.org/uniprot/P10845). Three amino acid residues, 262, 266 and 366, are important for the enzymatic activity. Thus, they should not be mutated (Rigoni et al., 2001). The sequence was saved in the Fasta format, and the new 3D structure of the protein from the RCSB PDB was stored for further studies.

\subsection{Immuno-informatic analyses}

\subsubsection{Linear B-cell epitopes prediction}

Several online tools were used to predict the linear B-cell epitopes. BepiPred-2.0 (http://www.cbs.dtu.dk/services/
BepiPred/) (Jespersen, Peters, Nielsen, \& Marcatili, 2017) was used to predict epitopes based on random forest algorithm trained on epitopes amino acids from the crystal structure of protein. Threshold of 0.5 and sensitivity of 0.6 were employed. Additionally, Bepipred server, accessed from IEDB (http://tools.immuneepitope.org/bcell/), was used to predict the linear B-cell epitopes by using hidden Markov model and propensity scale method (Larsen, Lund, \& Nielsen, 2006). The threshold of 0.35 with the sensitivity of 0.49 and the specificity of 0.75 was employed.

SVMTriP (http://sysbio.unl.edu/SVMTriP/) (Yao et al., 2012) was used to predict linear B-cell epitopes that integrate Tripeptide similarity and propensity score utilizing support vector machine (SVM) algorithm. Another tool was used to predict the linear epitopes of B-cell (http://imed.med.ucm.es/ Tools/antigenic.html) and to evaluate the occurrences of amino acids that acted as epitope in protein experimentally (Kolaskar \& Tongaonkar, 1990). Briefly, the average propensity of central amino acid residue of every 7-mer was evaluated and compared with the average propensity of the whole protein. The threshold to consider an antigenic amino acid residue was 1.0. Then, every 8-mer where all residues were above the threshold is considered as an epitope. ABCpred (http://crdd.osdd.net/raghava/abcpred/) was employed to predict continuous B-cell epitopes based on machine learning techniques, such as feed-forward (FNN) and recurrent neural network (RNN). As many as 20 mers could be predicted as epitope (Saha \& Raghava, 2006). The score used for the threshold was 0.81 .

\subsubsection{Conformational B-cell epitopes prediction}

Discotope $2.0 \quad$ (http://www.cbs.dtu.dk/services/DiscoTope/) was used to predict conformational B-cell epitopes from the calculation of surface accessibility and novel propensity amino acid score (Kringelum et al., 2012). A threshold of -1 with $30 \%$ sensitivity and $85 \%$ specificity was used. CBTOPE (Conformational B-cell Epitope Prediction) (http://crdd.osdd. net/raghava/cbtope/) server was also employed with the accuracy of about $84 \%$. This tool used SVM algorithm to train the program with the amino acid composition and physicochemical profile to predict the epitopes (Ansari \& Raghava, 2010). Threshold of -0.3 was used, and residues with the scale above 5 were considered epitopes. Moreover, exposed surface was analyzed with Emini surface accessibility which was accessed at IEDB Analysis Resource (Emini, Hughes, Perlow, \& Boger, 1985). Score of surface probability that resulted above one was predicted to be accessible on the surface of the protein.

\subsubsection{Examination of the conserved region}

Entropy method provided by Swiss Model ExPASy was used to determine the conserved region of the protein (Arnold, Bordoli, Kopp, \& Schwede, 2006; Benkert, Biasini, \& Schwede, 2011; Biasini et al., 2014). Entropy score below 2 is considered conserved residue which will be excluded from mutagenesis analysis. 

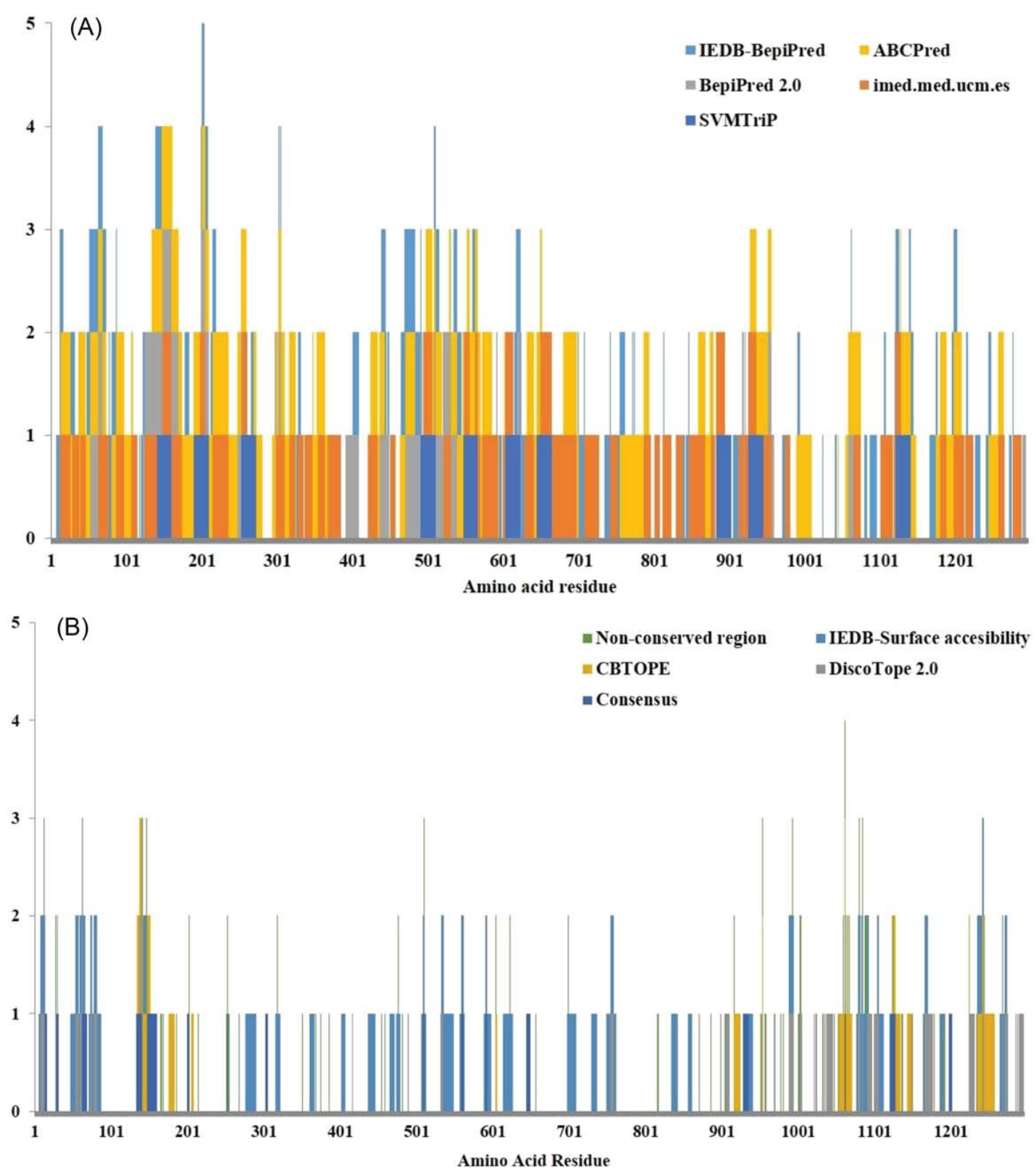

Figure 1. Graphical illustration of linear B-cell epitopes (A) and overlap of consensus from the linear B-cell epitopes with the conformational B-cell epitopes and non-conserved region $(B)$ to determine which residues are allowed to mutate.

\subsubsection{In silico mutagenesis of the predicted residues}

In silico mutagenesis and stability examination of the mutein were done using I-Mutant 2.0 (http://folding.biofold.org/imutant/i-mutant2.0.html) for every single substitution mutation of the determined residues (Capriotti, Fariselli, \& Casadio, 2005). The condition for the mutation was $\mathrm{pH} 7.4$ at the temperature of $37^{\circ} \mathrm{C}$, which conforms to human physiological condition. Mutation was allowed if the difference of the Gibbs free energy was positive which indicated an increase of protein stability. The difference of the Gibbs free energy was measured with the formula of $\Delta G$ (mutein) $\Delta G$ (wild-type protein). The residue that did not contain any positive value of difference of the Gibbs free energy was tried for deletion-type mutation. The proteins with mutated residues were subjected to the B-cell epitopic probability with VaxiJen (http://www.ddg-pharmfac.net/vaxijen/VaxiJen/
VaxiJen.html). This tool used auto cross-covariance pre-processing of protein while detecting antigenicity of residues with the help of $z$-descriptors as physicochemical parameters (Doytchinova \& Flower, 2007).

\subsection{Immuno-informatic analyses}

\subsubsection{D Modelling of mutated protein and validation} 3D protein was predicted and evaluated by PHYRE2 Protein Fold Recognition Server (http://www.sbg.bio.ic.ac.uk/phyre2). This tool displays the accuracy comparable to the widely used web servers for protein modelling, such as I-TASSER or Swiss-Model, but it is easier to use. PHYRE2 also provided investigator tools for 3D model evaluation and validation (Kelley, Mezulis, Yates, Wass, \& Sternberg, 2015). The 
Table 1. Analysis of substitution mutation stability with difference in Gibbs free energy method by I-Mutant 2.0 Condition of mutation was set at $\mathrm{pH}$ of 7.4 and temperature of $37^{\circ} \mathrm{C}$.

\begin{tabular}{lccccccc}
\hline$\Delta \Delta G$ & E147 & E510 & T1062 & E1080 & D1088 & N1089 & Q1090 \\
\hline V & -0.98 & -0.54 & -0.47 & 0.73 & -1.13 & -1.23 & -1.50 \\
L & -1.18 & -0.50 & -0.88 & 0.98 & -1.25 & -0.62 & -1.21 \\
I & -0.71 & -0.04 & -0.12 & 1.31 & -0.81 & -0.04 & -1.24 \\
M & -0.69 & -0.01 & 0 & 1.01 & -0.74 & 0.01 & -1.76 \\
F & -0.20 & 0.86 & 0.29 & 2 & -0.13 & -0.17 & -0.71 \\
W & -0.89 & -0.07 & -0.03 & 1.06 & -1.21 & -0.87 & -1.33 \\
Y & -1.60 & -0.28 & 0.47 & 1.25 & -0.47 & -0.63 & -1.52 \\
G & -2.05 & -1.15 & -1.20 & 0.13 & -1.51 & -0.91 & -1.92 \\
A & -2.12 & -1.17 & -0.54 & -0.13 & -2 & -1.61 & -2.78 \\
P & -2.48 & -2.14 & -1.31 & -0.96 & -2.50 & -2.05 & -2.87 \\
S & -1.74 & -0.66 & -0.09 & 0.06 & -1.06 & -1.12 & -2.03 \\
T & -1.29 & -0.18 & & 0.81 & -1.2 & -0.86 & -1.58 \\
C & -0.65 & -0.29 & -0.46 & 0.3 & -0.77 & -0.45 & -1.59 \\
H & -2.15 & -1.19 & -1.42 & -0.48 & -0.74 & -1.46 & -2.31 \\
R & -1.69 & -0.25 & 0.65 & 0.68 & -1.23 & -0.44 & -2.01 \\
K & -2.37 & -1.42 & -0.30 & -0.55 & -1.39 & -2.07 & -2.66 \\
Q & -1.78 & -0.94 & -0.69 & 0.26 & -1.12 & -1.07 & \\
E & & & 0.11 & & -1.06 & -0.68 & -1.84 \\
N & -2.62 & -1.24 & -0.31 & 0.26 & -1.45 & & -3.11 \\
D & -1.75 & -0.58 & 0.33 & 0.49 & & -0.89 & -1.74 \\
\hline
\end{tabular}

The bold values indicated the positive values of $\Delta \Delta G$.

evaluations of 3D protein quality provided by PHYRE2 were ProQ2 quality assessment, HHsearch alignment confidence, analysis of clashes, rotamers and disorder analysis based on Disopred. ProtParam was also used to analyze all protein properties and stability (Gasteiger et al., 2005). Ramachandran plot, however, was analyzed by RAMPAGE (http://mordred.bioc.cam.ac.uk/ rapper/rampage.php) ProSAweb (https://prosa.services.came.sbg.ac.at/prosa.php) was employed to evaluate the $3 D$ protein quality in terms of $Z$ score and residue energies plot. A good model is indicated with a Z-score inside the range characteristics of scores from other native proteins with similar sizes and groups, while the energy plot shows the local model quality for each central residue in every 40-mer fragment (Wiederstein \& Sippl, 2007). In addition, the protein model quality was also analyzed with VERIFY3D (Lüthy, Bowie, \& Eisenberg, 1992; Bowie, Lüthy, \& Eisenberg, 1991) and ERRAT (Colovos \& Yeates, 1993) from SAVES 5.0 web servers (http://servicesn.mbi.ucla. edu/SAVES/).

\subsubsection{Molecular dynamics (MD) simulation}

The 3D models of mutein generated and validated from PHYRE2 were subjected to MD simulation at MDweb (http:// mmb.irbbarcelona.org/MDWeb/) (Hospital et al., 2012). Gromacs Full MD setup with Gromos $53 a 6$ force field was employed. The system was solvated with TIP3P water molecules with a spacing distance of $15 \AA$ around the protein. $\mathrm{Na}$ + and $\mathrm{Cl}$ - were added to neutralize the system until the concentration reached $50 \mathrm{mM}$. Initially, heavy atoms were restrained with a force constant of $500 \mathrm{KJ} / \mathrm{mol} \times \mathrm{nm}^{2}$. The system was further equilibrated under NPT and NVT ensembles. The solvent was heated to $300 \mathrm{~K}$, and the force for restraining the heavy atoms was gradually diminished until the simulation was done without restraint. The temperature and pressure were kept at default with velocity-rescale and Parinello-Rahman algorithms, respectively.
The root-mean-square deviation (RMSD) aligned through trajectory, root-mean-square fluctuation (RMSF) of all residues, the radius of gyration $(\mathrm{Rg})$, solvent-accessible surface area (SASA) and secondary structure projection aligned through trajectory for each protein were computed. SASA was measured with StrucTools (https://hpcwebapps.cit.nih. gov/structbio/basic.html) with an MSMS program (Michel Sanner's Molecular Surface). The surface probe size was 1.4 $\AA$, and the surface of only atom in general 20 amino acids was calculated. The same tool was used to calculate which residues of the protein contributed to the following secondary structure: $\alpha$-helix, $\beta$-strand, $\beta$-turn, $\beta$-bridge, random coil and 310-helix. The secondary structure projection plot was built by calculating the amount of residue that composed each secondary structure types within the simulation time. Intramolecular hydrogen bonds were analyzed by first removing all water molecules and ions from the trajectory. Hydrogen bond calculation tool (http://cib.cf.ocha.ac.jp/ bitool/HBOND/) was employed to calculate the amount of hydrogen bond from each pdb file. The MD was done in 26 snapshots in which each snapshot was $0.5 \mathrm{~ns}$. The total time for MD simulation was 13,000 ps.

\section{Results and discussion}

BoNT/A market is globally diverse at the moment, with one industry producing its own formulation and protein modification for its novelty (Dressler, 2016). Therefore, it is interesting to develop the low antigenicity type of this protein since this protein is a foreign one. In silico method provides an initial study for mutagenesis to suggest which amino acid residue should be mutated to show lower antigenicity while still retaining its stability and activity compared to the native protein.

\subsection{B-cell epitopes prediction}

Various tools are available in a lot of web-server software to predict antigenicity of a protein, with regard to the previously known 3D structure of the BoNT/A. BoNT/A has been characterized with X-ray crystallography with multiple isomorphous replacement as phasing approach (Lacy et al., 1998). The downloaded PDB files (3BTA) were directly used for the determination of B-cell epitopes. The study, thus, can be directed to the determination of the B-cell epitopes.

Some researchers used web-server tools to evaluate the antigenicity of protein residues (Fattahian, Riahi-Madvar, Mirzaee, Asadikaram \& Rahbar, 2017; Zarei et al., 2018). In total, five tools were employed to predict the linear B-cell conformation, and three types of web-server software were used to predict the conformational B-cell epitopes with various amino acid lengths. The residues obtained from the linear B-cell epitopes prediction must comply with at least three of the five tools. The consensus residues obtained using this method were overlaid with the result of the prediction of the conformational B-cell epitopes and the nonconserve region to select the epitopes which are permitted to be mutated. The selected epitopes can be seen 
Table 2. Deletion of the determined residues with no increase of $\Delta \Delta G$ value.

\begin{tabular}{lllllll}
\hline & $\Delta E 147$ & $\Delta D 1088$ & $\Delta Q 1090$ & $\Delta E 147 \Delta D 1088$ & $\Delta E 147 \Delta Q 1090$ & $\Delta E 147 \Delta D 1088 \Delta Q 1090$ \\
\hline VaxiJen Score & 0.3929 & 0.3915 & 0.3906 & 0.391 & 0.3901 & 0.3903 \\
\hline
\end{tabular}

The initial VaxiJen score of the BoNT/A is 0.3934.

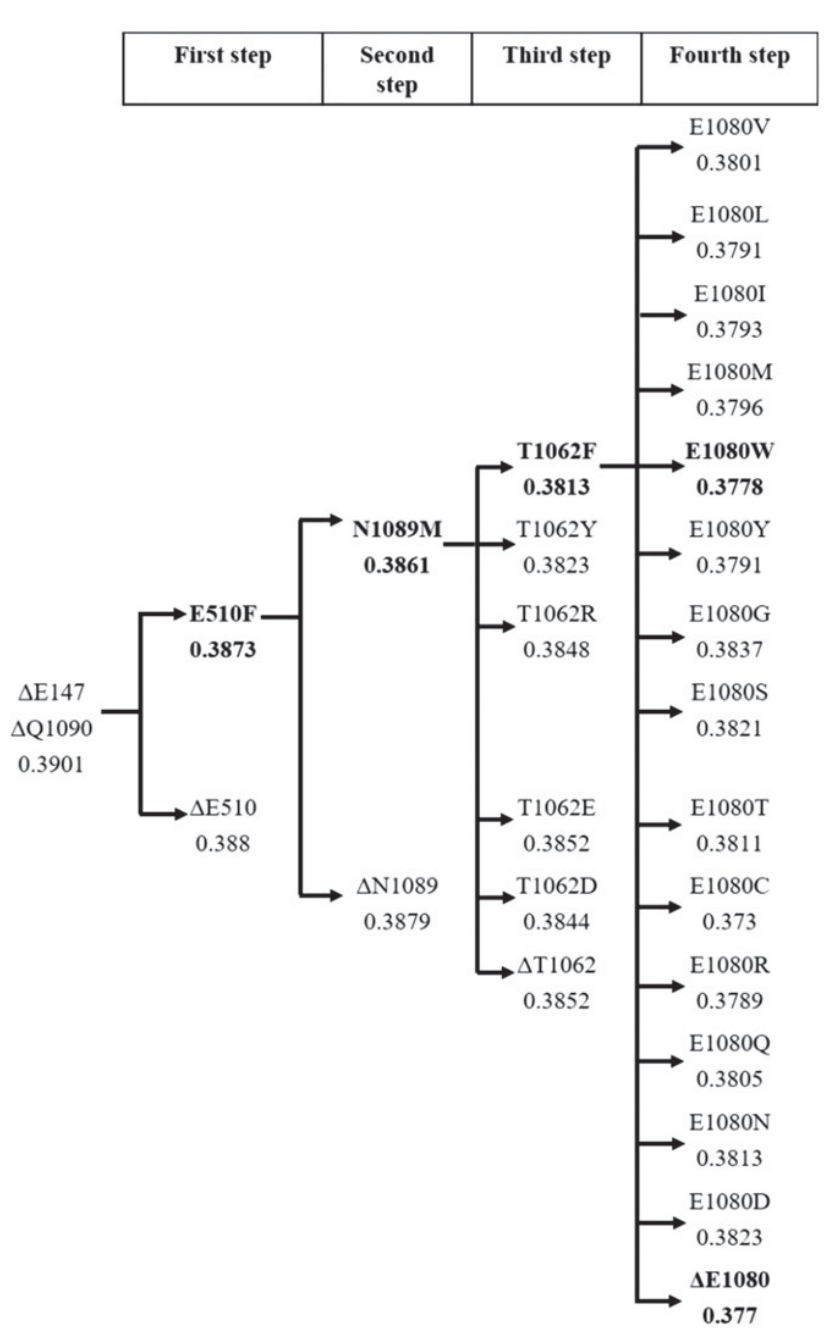

Figure 2. Sequential substitution in silico mutagenesis strategy flowchart with the help of the reduction of immunogenicity score from VaxiJen. The mutation was retained after each step, while the initial protein used the result of the deletion mutation indicated in bold.

schematically in Figure 1. The same tools can also be used to predict the antigenicity of the protein directed to vaccine design of emerging pathogens (Bazmara et al., 2017; Hajighahramani et al., 2017; Kalyanaraman, 2018; Negahdaripour et al., 2017; Nezafat et al., 2016; Pahil, Taneja, Ansari, \& Raghava, 2017; Shi et al., 2015; Yasmin et al., 2016).

Conserved region determined by entropy method (Supplementary data 1) usually contributed to the structural and functional properties. This region was, therefore, excluded in order to ensure the stability and the conformation of the muteins (Zarei et al., 2018). Based on the linear B-cell epitopes, conformational B-cell epitopes, surface accessibility and conserved region prediction, seven residues were obtained out of 1296 amino acids residue. T1062 was the overlapping region with consensus linear epitopes and two other conformational residues, whereas E147, E510, E1080,
D1088, N1089 and Q1090 were obtained from the overlapping process of consensus linear epitopes and one of the Bcell conformational epitopes prediction tools. Indeed, most polar amino acids were predicted as B-cell epitopes since these amino acids tend to be at the surface of a protein that can be recognized by immune system cells and antibody (Swearingen et al., 2016). While referring to DiscoTope 2.0 conformational B-cell prediction, many of the residues were located near the $N$-terminal (8-141) and C-terminal (906-1296) which were normally exposed (Sefid, Rasooli, Jahangiri, \& Bazmara, 2015).

\subsection{In silico mutagenesis of BoNT/A and $3 D$ modelling and validation of the muteins}

I-Mutant 2.0 is able to mutate the candidate amino acid with the 19 other amino acids. Table 1 summarizes the difference in Gibbs free energy after a substitution mutation. Positive value indicated that the residue is allowed to mutate into the specified amino acid, correlating with an increase in stability after the protein has been mutated due to the positive Gibbs free energy.

In general, the strategies for the in silico mutagenesis started from the deletion analysis with the amino acids that did not contain any positive values from the result of 1 Mutant 2.0 analysis. Table 2 shows the result of the combination analysis with the help from VaxiJen for the immunogenicity score and bacteria as the source organism. Although the deletion at D1088 alone reduced the immunogenicity score, the combined score of all three deletions was still higher than when the deletions were only at E147 and Q1090. Then, mutagenesis strategy was continued with the serial mutation with the other amino acids with positive content of $\Delta \Delta G$. The VaxiJen score can be seen in Figure 2 .

In summary, there were two proposed mutations. Option one was $\Delta E 147, \mathrm{E} 510 \mathrm{~F}, \mathrm{~T} 1062 \mathrm{~F}, \Delta E 1080, \mathrm{~N} 1089 \mathrm{M}, \Delta Q 1090$ with a VaxiJen score of 0.377 . Option two was $\triangle E 147, E 510 \mathrm{~F}$, T1062F, E1080W, N1089M, $\Delta Q 1090$ with a VaxiJen score of 0.3778 . Both proposed muteins generated the two lowest VaxiJen scores which were analyzed from the total sequence. Changing the immunogenic residue into non-polar amino acids resulted in the less-immunogenicity protein (Zarei et al., 2018). One hotspot residue showed by IEDB with a good surface accessibility promoting mutability was residue 1079-1092. This residue was also separately analyzed with the VaxiJen. Both muteins showed lower VaxiJen score in the hotspot residue, but option two revealed a lower VaxiJen score than option one (Table 3).

These two proposed muteins were subjected to 3D protein modelling and continued with the validation of the analysis together with the native protein (Figure 3). Protein model validation that was initially analyzed by various PHYRE2 investigators revealed no major difference between 
Table 3. Immunogenic analysis by VaxiJen of the muteins, both total protein sequence and the selected hotspot residue (1079-1092).

\begin{tabular}{llll}
\hline Native & Epitope (1079-1092) & VaxiJen score of protein & VaxiJen score of surface accessibility \\
\hline Native protein & LNEKEIKDLYDNQS & $\mathbf{0 . 3 9 3 4}$ & 0.5113 \\
First option $(\triangle E 147, \mathrm{E} 510 \mathrm{~F}, \mathrm{~T} 1062 \mathrm{~F}, \Delta E 1080, \mathrm{~N} 1089 \mathrm{M}, \Delta Q 1090)$ & LNKEIKDLYDMS & 0.377 & 0.3388 \\
Second option $(\Delta E 147, \mathrm{E} 510 \mathrm{~F}, \mathrm{~T} 1062 \mathrm{~F}, \mathrm{E} 1080 \mathrm{~W}, \mathrm{~N} 1089 \mathrm{M}, \Delta Q 1090)$ & LNWKEIKDLYDMS & 0.3778 & 0.1276 \\
\hline
\end{tabular}

Bold values signifies the native protein hotspot segment vaxijen score and compare (and emphasize) the reduction of the value of the mutein.
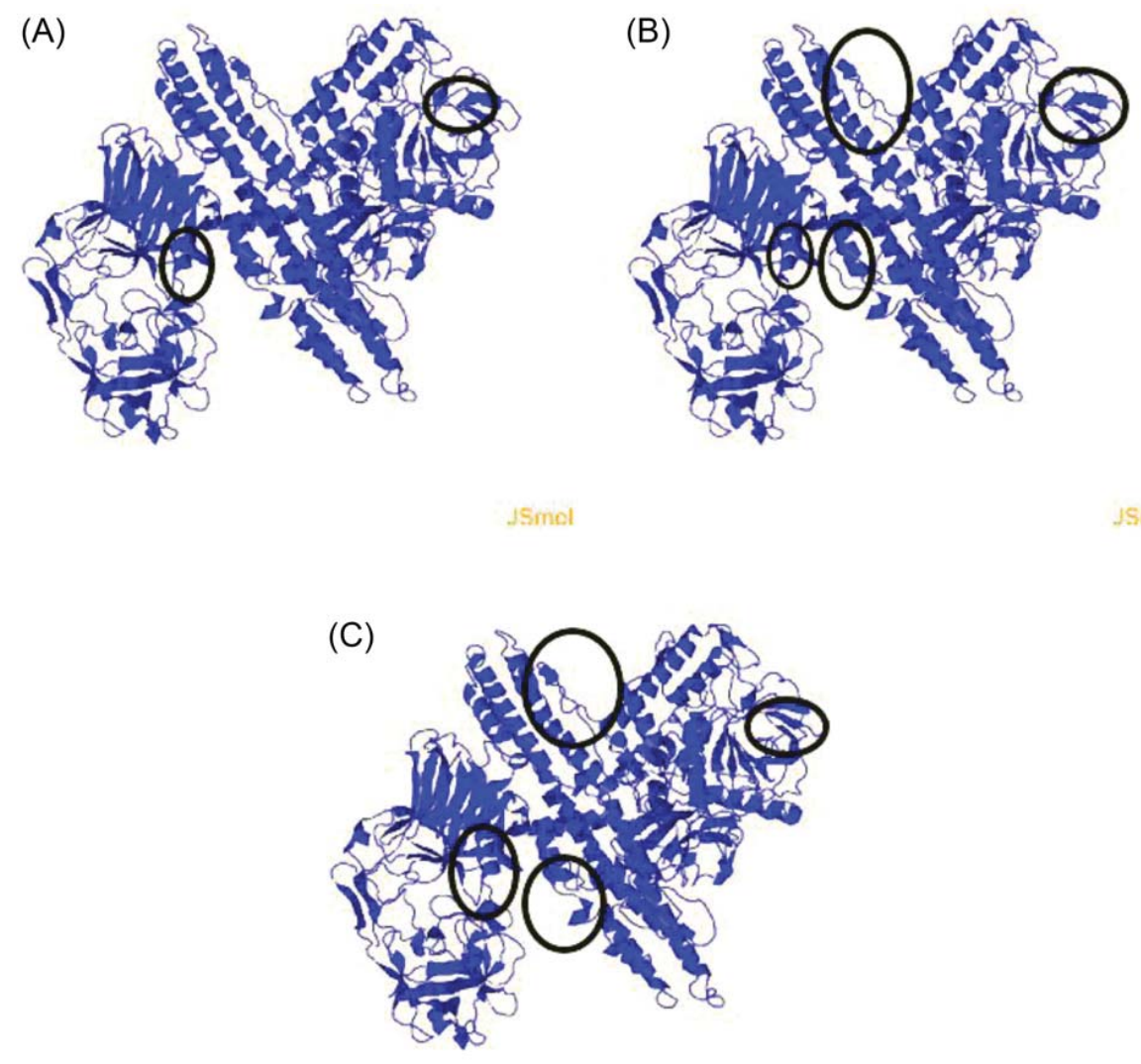

jSmol

Figure 3. 3D modelling of the first-option mutein (A), second-option mutein (B) and native BoNT/A (C) built by PHYRE2. The area indicated with circle is the one that contains difference in $3 \mathrm{D}$ structure with the native protein.

Table 4. Validation of both mutein 3D models built by PHYRE2.

\begin{tabular}{lccc}
\hline & & First-option mutein $(\Delta E 147, E 510 F$, & Second-option mutein $(\Delta E 147, E 510 F$, \\
& Native BoNT/A & T1062F, $\Delta E 1080, N 1089 \mathrm{M}, \Delta Q 1090)$ & T1062F, E1080W, N1089M, $\Delta Q 1090)$ \\
\hline Ramachandran analysis: & & & $89.7 \%$ \\
Favoured region & $81.9 \%$ & $89.7 \%$ & $7.8 \%$ \\
Allowed region & $13.4 \%$ & $8 \%$ & $2.5 \%$ \\
Outlier region & $4.7 \%$ & $2.3 \%$ & -13.82 \\
Z-Score of overall model quality & -13.81 & -13.51 & $80.82 \%$ (pass) \\
Average 3D-1D score & $83.79 \%$ (pass) & $81.12 \%$ (pass) & 85.7831 \\
ERRAT overall quality factor A & 72.7273 & 85.7831 & 80.8081 \\
ERRAT overall quality factor B & 69.7789 & 80.9886 & \\
\hline
\end{tabular}

the mutein and native protein (Supplementary Data 2). Further validation analyses can be seen in Table 4 .

Both Ramachandran plot analyses of the mutein yield lower residual values for the outlier region compared to those of the native BoNT/A. The $z$-score from the PROSAweb was similar to the QMean from the SWISS-MODEL, indicating similarity of the 3D model to the experimental protein with similar size (Benkert, Tosatto, \& Schomburg, 2008). The $z-$ score of the first-option mutein was within the range of the other native protein with similar size, which indicated an appropriate model (Figure $4(B))$. Both the $z$-score of the native protein and the second-option mutein were not in the range in the plot (Figure $4(A, C)$ ). However, the $z$-score of the second-option mutein was closer to that of the native BoNT/A protein. Due to its closeness in its $z$-score to the native BoNT/A, the second-option mutein model can also be considered as valid. The local energy plots of each residue were similar to the 3D model protein, and these positive values of the energies could be found in the native protein as well (Figure 4(D-F)). VERIFY3D compared the 3D model with the amino acid sequence (1D) and its environment (Eisenberg, Lüthy, \& Bowie, 1997). All the 3D protein models 
(A) 2.Scose: -13.81

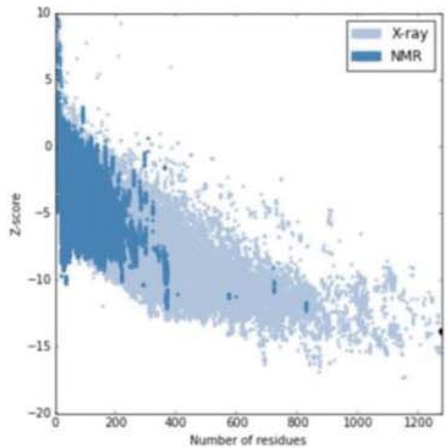

(D)

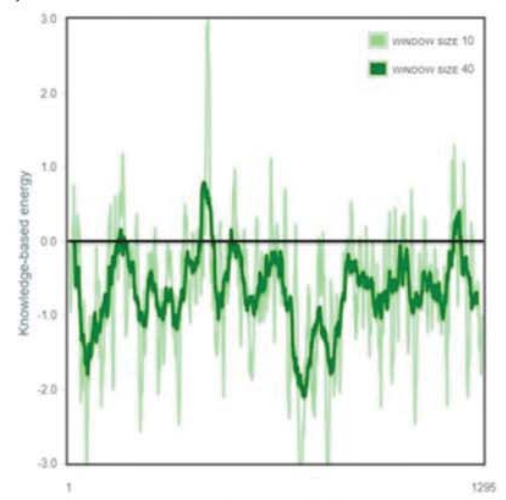

(B)

$$
\begin{aligned}
& \text { Overall model quality } \\
& \text { Z-Score: }-13.51
\end{aligned}
$$

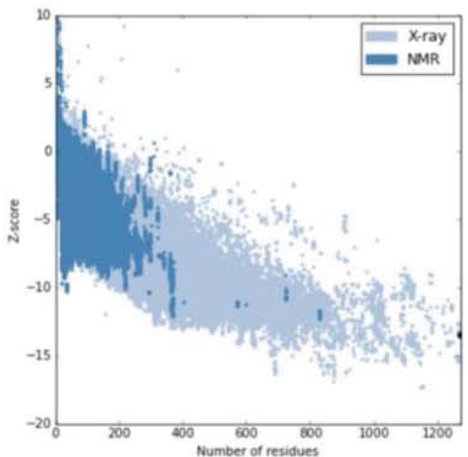

(E)

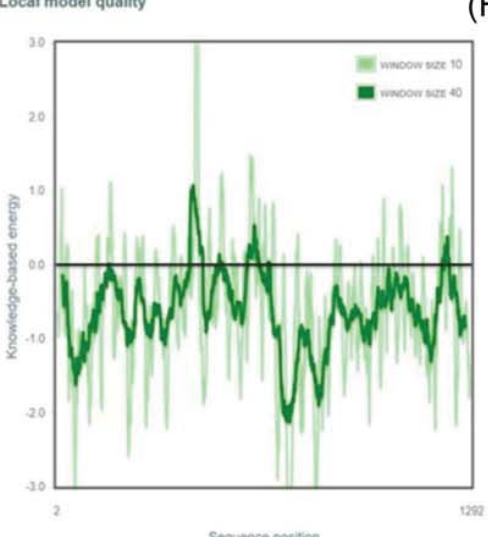

(C)

$$
\begin{aligned}
& \text { Overall model quality } \\
& z-\text {-Score: } \\
& -13.82
\end{aligned}
$$

Sequencespostion

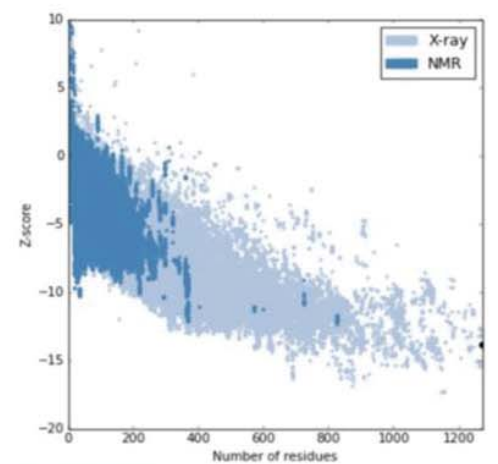

(F)

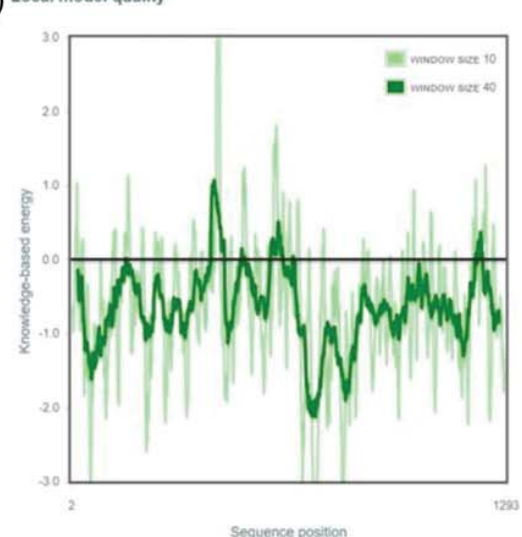

Figure 4. 3D model validation by PROSAweb. (A-C) Overall model quality with the use of $z$-score of the native BoNT/A (A), first-option mutein (B) and second-

\begin{tabular}{|c|c|c|c|}
\hline & Native BoNT/A & $\begin{array}{l}\text { First-option mutein }(\Delta E 147, \text { E510F, } \\
\mathrm{T} 1062 \mathrm{~F}, \Delta E 1080, \mathrm{~N} 1089 \mathrm{M}, \Delta Q 1090)\end{array}$ & $\begin{array}{l}\text { Second-option mutein }(\Delta E 147, \text { E510F, } \\
\text { T1062F, E1080W, N1089M, } \Delta Q 1090)\end{array}$ \\
\hline Isoelectric point & 6.05 & 6.31 & 6.31 \\
\hline Molecular weight & 149453.89 & 149148.75 & 149334.97 \\
\hline Instability index ${ }^{a}$ & 29.35 & 29.33 & 29.05 \\
\hline Aliphatic index ${ }^{b}$ & 90.24 & 90.45 & 90.38 \\
\hline $\begin{array}{l}\text { Grand average of hydropathic- } \\
\text { ity }(\text { GRAVY) }\end{array}$ & -0.366 & -0.347 & -0.347 \\
\hline Estimated half-life $^{d}$ & $>30 \mathrm{~h}$ & $>30 \mathrm{~h}$ & $>30 \mathrm{~h}$ \\
\hline
\end{tabular}
option mutein (C). The black dot indicated the (D-F) comparison of the residue energy plot for the local model quality for every 10 and 40 residues windows for (D) native BoNT/A, (E) first-option mutein and (F) second-option mutein.

Table 5. Native BoNT/A and both mutein properties, stability and hydrophilicity analysis.

${ }^{\text {a }}$ The protein is considered stable if the instability index is below 40 .

${ }^{\mathrm{b}}$ The protein is considered stable if the aliphatic index is more positive.

'Protein is considered more hydrophobic if the value is more positive.

${ }^{\mathrm{d}}$ The half-life of the protein was predicted using mammalian reticulocytes as model in vivo.

Table 6. Average values of RMSD, $\mathrm{R}_{\mathrm{g}}$ and RMSF for the native BoNT/A and both muteins.

\begin{tabular}{lccc}
\hline & & First-option mutein $(\Delta E 147$, E510F, & Second-option mutein $(\Delta E 147$, E510F, \\
& Native BoNT/A & T1062F, $\Delta E 1080, N 1089 \mathrm{M}, \Delta Q 1090)$ & T1062F, E1080W, N1089M, $\Delta Q 1090)$ \\
\hline RMSD & $9.4649 \AA$ & $9.4564 \AA$ & $9.4552 \AA$ \\
$\mathrm{R}_{\mathrm{g}}$ & $7.6025 \AA$ & $7.5973 \AA$ & $7.5915 \AA$ \\
SASA & $501.814 \mathrm{~nm}^{2}$ & $508.462 \mathrm{~nm}^{2}$ & $502.185 \mathrm{~nm}^{2}$ \\
RMSF & $0.3783 \AA$ & $0.3833 \AA$ & $0.3892 \AA$ \\
\hline
\end{tabular}

yield a result of more than $80 \%$ from its residues with a 3D-1D score $>=0.2$ (Table 4). The non-bonded interactions from the six combinations of Carbon (C), Nitrogen (N) and Oxygen (O) and plotted the error function from every nineresidue sliding windows were analyzed using ERRAT (Colovos \& Yeates, 1993). The ERRAT score of both muteins was higher than that of the native BoNT/A. Overall, these validation results indicated that the $3 \mathrm{D}$ model of the mutein was proper.

Although the mutated residues were ensured in the nonconserved region, the stability of the protein must still be evaluated. ProtParam provided some tools to analyze protein 

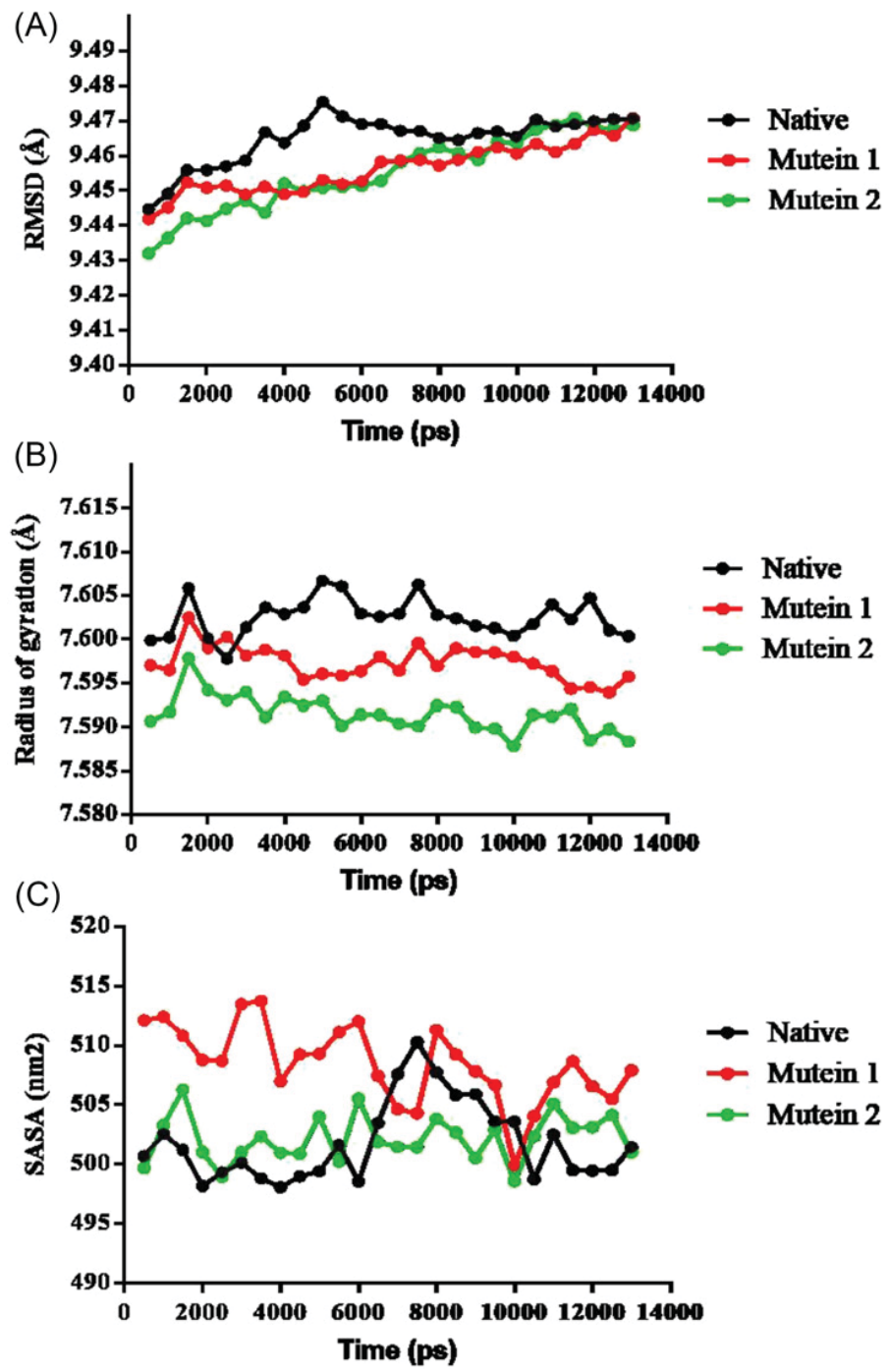

Figure 5. Time evolution of backbone RMSD (A), $R_{g}$ plot (B), and SASA (C) as a function of time for native protein, first-option mutein and second-option mutein.

properties along with the stability (Table 5). Based on the instability index and aliphatic index, both muteins were more stable than the native protein. According to the hydropathy analysis by GRAVY, both muteins had higher scores which signify a more hydrophobic nature than the native, suggesting the lower number of residues on the surface and thus reducing antigenicity (Shi et al., 2015). Instability index led to empiric experiment and algorithm, while aliphatic index was more related to the thermostability of protein due to the hydrophobic stabilization (Ikai, 1980). In this case, the aliphatic index implied that the first-option mutein had better thermostability compared to the other two proteins.

\subsection{MD simulation}

MD analysis is one of the most frequently used methods to determine protein stability in solvated condition. Gromos $53 a 6$ force field was reported to give a better agreement with wet-lab work for Histatin 5 (Henriques, Cragnell, \& Skepö, 2015). Generally, RMSD of the protein backbone can be plotted against time to predict stability. The RMSD plot can be seen in Figure 5(A). A similar trend of the sudden increment of deviation from the beginning of the simulation until the time period of $2.5 \mathrm{~ns}$ also occurred. There was a sharp increase of RMSD for the native BoNT/A in the time period of $5 \mathrm{~ns}$, which reached $\sim 9.475 \AA$. The RMSD values of the native protein reached stability after $6 \mathrm{~ns}$, in which the RMSD values ranged from $\sim 9.465$ to $\sim 9.47 \AA$. Until 10 ns of simulation time, the RMSD values of both muteins were lower than those of the native one. The RMSD values of the muteins gradually increased in a small difference until the end of the simulation. This small difference in the fluctuations after the relaxation period led to stable trajectories in the simulation. The two muteins showed more deviations from the initial structure. However, the deviation of the native protein was only a maximum of $5 \mathrm{~ns}$. At the end of the RMSD simulation, the RMSD values of the muteins and native protein were similar, though. The values were about 4.7 $\AA$ for both native BoNT/A and the first-option mutein, and $4.68 \AA$ for the second-option mutein.

The $R_{g}$ is the overall size of the protein, computed by measuring all the mass-weight root-mean-square distance from all atoms from the centre of the mass (Rajendran \& Sethumadhavan, 2014). The average $R_{g}$ of both muteins was 

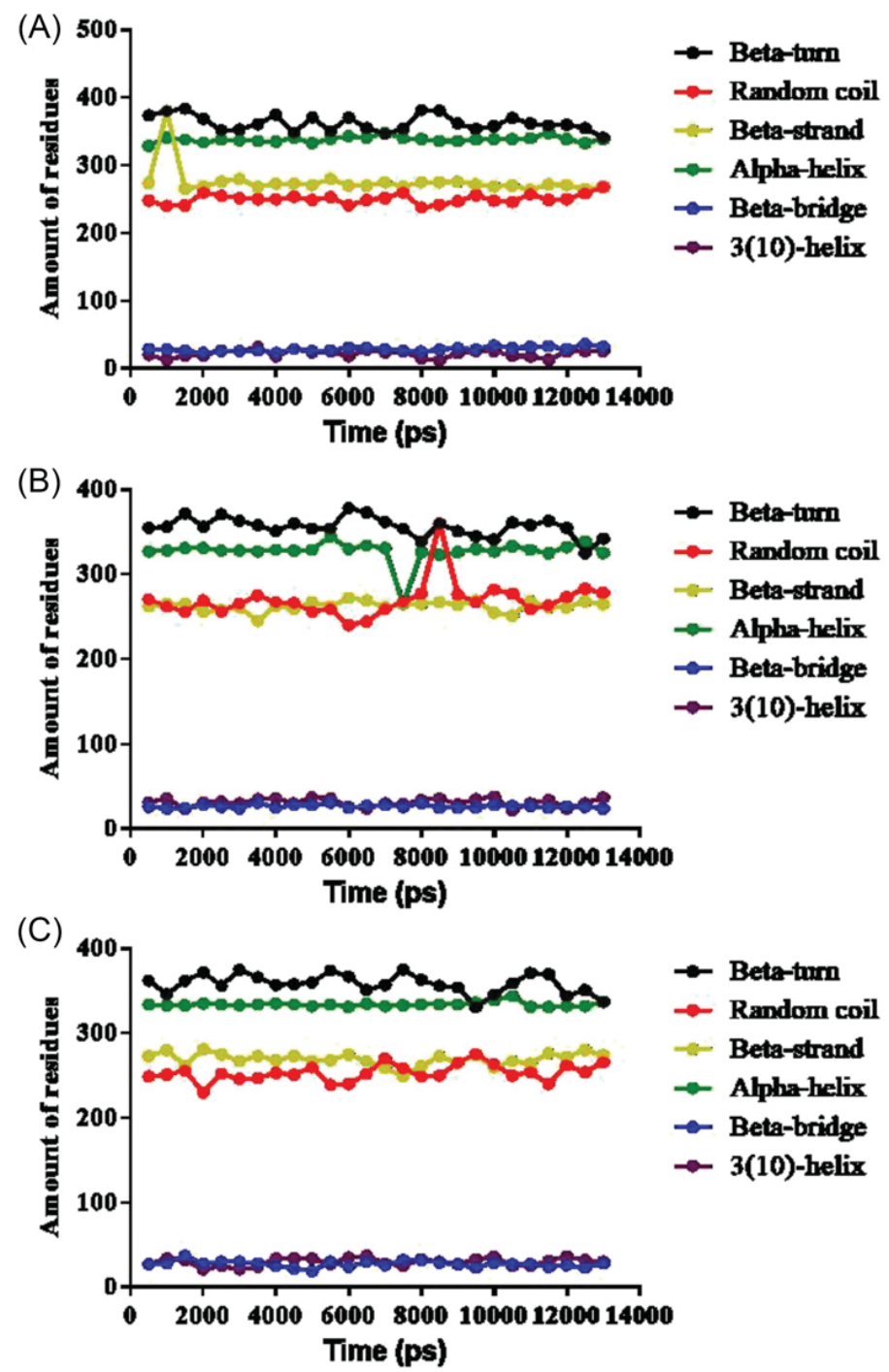

Figure 6. Time evolution of the amount of amino acids residue that contributed to secondary structure of the native BoNT/A (A), mutein first option (B) and mutein second option $(C)$

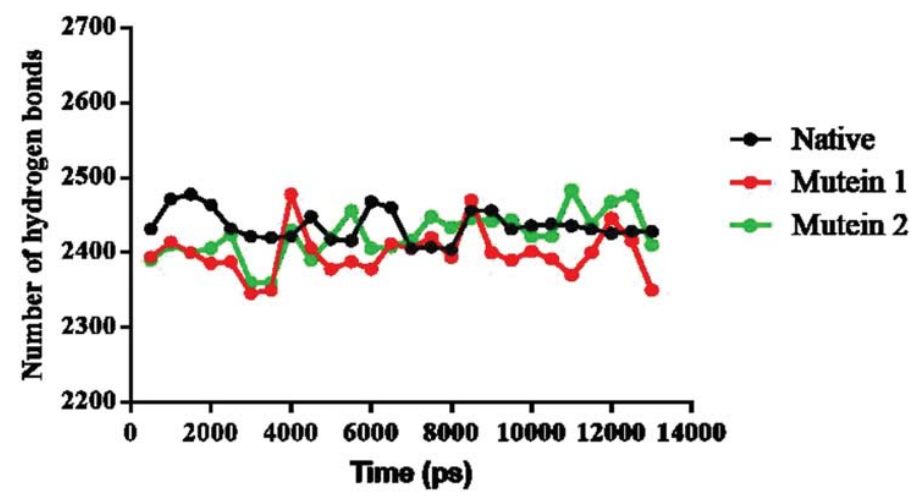

Figure 7. The number of protein intramolecular hydrogen bonds in the native and mutated BoNT/A vs. time at $300 \mathrm{~K}$.

smaller than the native BoNT/A, indicating more compactness of the mutant (Table 6). The range of $R_{g}$ for the native protein was between 7.59783 and $7.6067 \AA$. The range of the first-option mutein was between 7.59394 and $7.60253 \AA$ while the range of the second-option mutein was between 7.58789 and $7.59786 \AA$ (Figure 5(B)). Both muteins were more compact compared to the native protein. By counting the differential of the maximum and minimum $R_{g}$ of all proteins, the mutein in the second option was more flexible than the native, but the mutein in the first option was more rigid than the native one. Nonetheless, all the differences did not even reach $0.01 \AA$. 

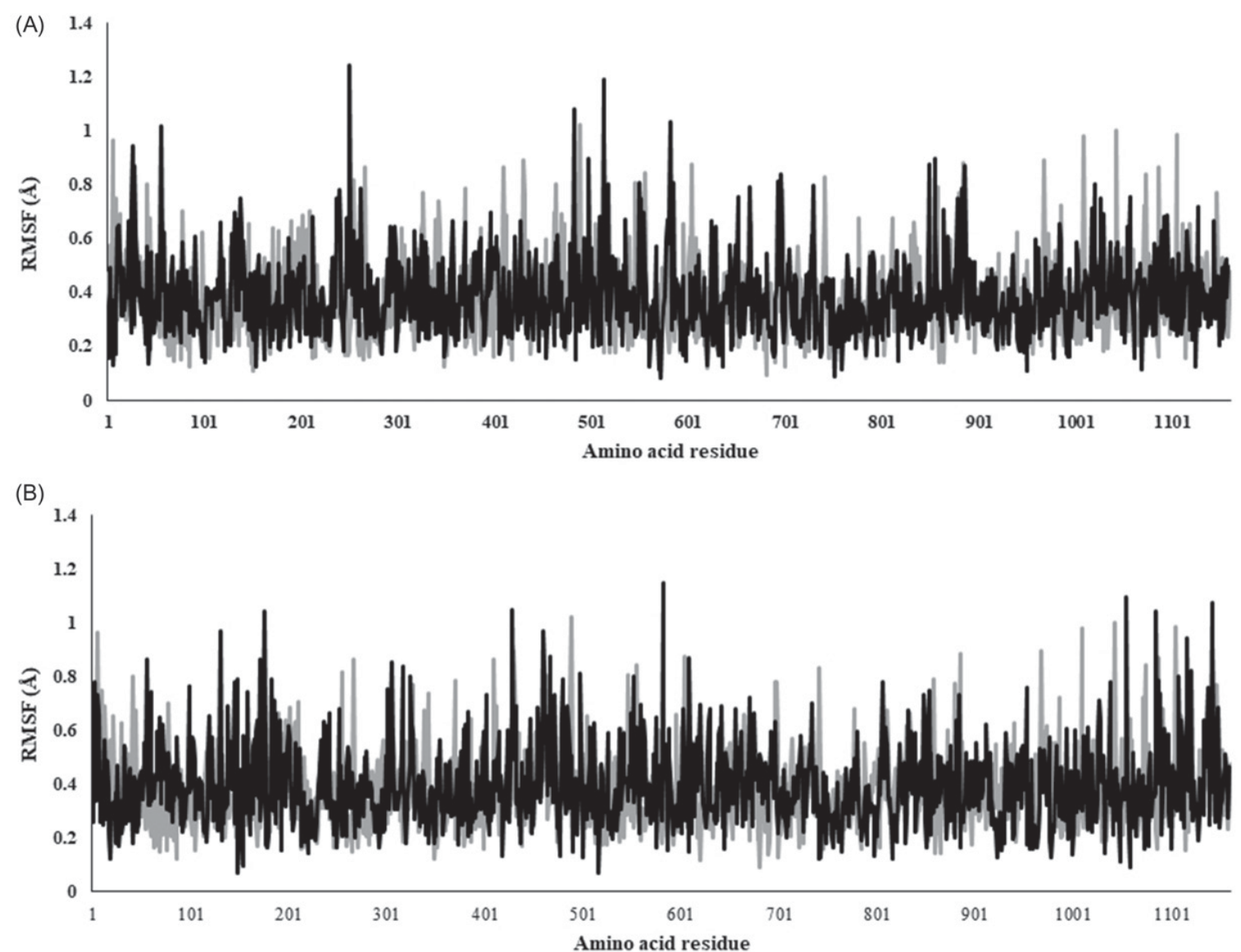

Figure 8. Comparison of the RMSF plot of each residue of (A) native BoNT/A (grey line) vs. first-option mutein (black line) and (B) native BoNT/A (grey line) vs. second-option mutein (black line).

Another parameter to measure the geometry of protein is SASA. The SASA value of native BoNT/A was comparatively similar to the second-option mutein, whereas the SASA value of the first-option mutein was higher than that of the native BoNT/A (Table 6). While the native BoNT/A and the firstoption mutein underwent a change in surface residues in the simulation period, the second-option mutein SASA value was relatively constant, indicating less transition of conformation (Figure $5(C)$ ). This fact was reinforced with the secondary structure prediction along the projection time (Figure 6). The second-option mutein showed less alteration in the secondary structure, implying more conformational stability.

Hydrogen bonds played an important role in assessing the stability of the protein. In the trajectory, the range of the amount of intramolecular hydrogen bonds for native was between 2404 and 2478 bonds, that of the first-option mutein was 2346-2478 bonds, and that of the second-option mutein was between 2360 and 2484 bonds. The fluctuation of intramolecular hydrogen bonds can be seen in Figure 7, where the native BoNT/A is apt to maintain its amount of hydrogen bonds during the simulation, indicating more rigidity compared to the two muteins.

The flexible regions were located with the help of RMSF of all residues. The RMSF values of native BoNT/A and the muteins were comparable (Figure 8) although both muteins gained more flexibility compared to the native protein (Table 6). Native BoNT/A and both muteins generated similar RMSF mean and deviation of $0.3783 \pm 0.1405 \AA$ for native BoNT/A, $0.3833 \pm 0.1442 \AA$ for the first-option mutein and $0.3892 \pm$ $0.1537 \AA$ for the second-option mutein. Nevertheless, all of the RMSF values were small and below $1 \mathrm{~nm}$, indicating stability of the protein (Nezafat et al., 2016). However, many of the RMSF values were above average, indicating flexibility, and, indeed, BoNT/A was a flexible protein (Silvaggi et al., 2007).

Based on the RMSD, RMSF Rg, SASA, secondary structure prediction and intramolecular hydrogen bond plot, it is evident that the mutation gave more flexibility to the protein in general. The difference was not large; thus, the gain of the flexibility did not disturb the functional behaviors of the protein (Kamaraj \& Purohit, 2013). The mutation did not exhibit destructive impacts to the protein orientation and the function since both BoNT/A mutation options were allowed to make the protein less immunogenic.

\section{Conclusion}

The in silico mutagenesis to generate less immune-reactive BoNT/A has been proposed. There were two proposed options. The first option was $\Delta E 147, \mathrm{E} 510 \mathrm{~F}, \mathrm{~T} 1062 \mathrm{~F}, \Delta E 1080$, $\mathrm{N} 1089 \mathrm{M}$, and $\Delta Q 1090$ which generate the lowest antigenicity score for the overall protein. The second one was $\Delta E 147$, E510F, T1062F, E1080W, N1089M, $\Delta Q 1090$ which was the second lowest antigenicity score for the overall protein. 
However, the antigenicity score for the hotspot residue of 1079-1092 was much lower than that of the first option. Both muteins showed better stability in relation to instability index, aliphatic index and hydropathy analysis compared to the native protein. The MD simulation indicated that the mutation is more flexible to the protein; thus, it would not disrupt the functionality of the protein. These suggestions can be used as rough guidelines for the experimental mutagenesis to save time and cost. In addition, the results of this study provide a breakthrough of better therapeutic and cosmetic suitability through the properties of its BoNT/ A muteins.

\section{Acknowledgements}

We thank Helen Hendaria Kamandhari, Ph.D., for her proofreading and comments.

\section{Disclosure statement}

No potential conflict of interest was reported by the authors.

\section{Author contributions}

Both SEET and YMV wrote the manuscript. Regarding the work collaboration, SEET conducted the mutagenesis, the 3D modelling and the stability analysis; YMV determined which consensus sequence to be mutated, the occurrence of the mutation (mutagenesis) and the analysis of the immunogenicity of the muteins; and SEDP supervised the work.

\section{Funding}

We would like to thank the Faculty of Biotechnology of Universitas Surabaya for the funding.

\section{References}

Ansari, H. R., \& Raghava, G. P. (2010). Identification of conformational Bcell epitopes in an antigen from its primary sequence. Immunome Research, 6(1), 6-9. doi:10.1186/1745-7580-6-6

Arnold, K., Bordoli, L., Kopp, J., \& Schwede, T. (2006). The SWISS-MODEL workspace: A web-based environment for protein structure homology modelling. Bioinformatics, 22(2), 195-201. doi:10.1093/bioinformatics/ bti770

Babiloni, A. H., Kapos, F. P., \& Nixdorf, D. R. (2016). Intraoral administration of botulinum toxin for trigeminal neuropathic pain. Oral Surgery, Oral Medicine, Oral Pathology and Oral Radiology, 121(6), e148-e153. doi:10.1016/j.00oo.2016.03.013

Bazmara, H., Rasooli, I., Jahangiri, A., Sefid, F., Astaneh, S. D. A., \& Payandeh, Z. (2017). Antigenic properties of iron regulated proteins in Acinetobacter baumannii: An in silico approach. International Journal of Peptide Research and Therapeutics, 1-9 [published online]. doi: 10.1007/s10989-017-9665-6

Benkert, P., Biasini, M., \& Schwede, T. (2011). Toward the estimation of the absolute quality of individual protein structure models. Bioinformatics, 27(3), 343-350. doi:10.1093/bioinformatics/btq662

Benkert, P., Tosatto, S. C., \& Schomburg, D. (2008). QMEAN: A comprehensive scoring function for model quality assessment. Proteins: Structure, Function, and Bioinformatics, 71(1), 261-277. doi:10.1002/ prot.21715

Bentivoglio, A. R., Del Grande, A., Petracca, M., lalongo, T., \& Ricciardi, L. (2015). Clinical differences between botulinum neurotoxin type $A$ and B. Toxicon, 107, 77-84. doi:10.1016/j.toxicon.2015.08.001
Biasini, M., Bienert, S., Waterhouse, A., Arnold, K., Studer, G., Schmidt, T., ... Schwede, T. (2014). SWISS-MODEL: Modelling protein tertiary and quaternary structure using evolutionary information. Nucleic Acids Research, 42(W1), W252-W258. doi:10.1093/nar/gku340

Bowie, J. U., Lüthy, R., \& Eisenberg, D. (1991). A method to identify protein sequences that fold into a known three-dimensional structure. Science, 253(5016), 164-170. doi:10.1126/science.1853201

Brooks, V. B. (1954). The action of botulinum toxin on motor-nerve filaments. The Journal of Physiology, 123(3), 501-515. doi:10.1113/ jphysiol.1954.sp005067

Capriotti, E., Fariselli, P., \& Casadio, R. (2005). I-Mutant2. 0: Predicting stability changes upon mutation from the protein sequence or structure. Nucleic Acids Research, 33(Web Server), W306-W310. doi:10.1093/nar/ gki375

Careta, M. F., Delgado, L., \& Patriota, R. (2015). Report of allergic reaction after application of botulinum toxin. Aesthetic Surgery Journal, 35(5), NP102-NP105. doi:10.1093/asj/sju105

Carruthers, A., \& Carruthers, J. (2005). Botulinum toxin type A. Journal of the American Academy of Dermatology, 53(2), 284-290. doi:10.1016/ j.jaad.2005.03.060

Castelao, M., Marques, R. E., Duarte, G. S., Rodrigues, F. B., Ferreira, J., Sampaio, C., ... Costa, J. (2017). Botulinum toxin type A therapy for cervical dystonia. Cochrane Database of Systematic Reviews, 12, CD003633. doi:10.1002/14651858.CD003633.pub3.

Colovos, C., \& Yeates, T. O. (1993). Verification of protein structures: Patterns of nonbonded atomic interactions. Protein Science, 2(9), 1511-1519. doi:10.1002/pro.5560020916

Doytchinova, I. A., \& Flower, D. R. (2007). VaxiJen: A server for prediction of protective antigens, tumour antigens and subunit vaccines. $B M C$ Bioinformatics, 8(4), 1-7. doi:10.1186/1471-2105-8-4

Dressler, D. (2016). Botulinum toxin drugs: Brief history and outlook. Journal of Neural Transmission, 123(3), 277-279. doi:10.1007/s00702015-1478-1

Dressler, D., \& Bigalke, H. (2017). Immunological aspects of botulinum toxin therapy. Expert Review of Neurotherapeutics, 17(5), 487-494. doi: 10.1080/14737175.2017.1262258

Eisenberg, D., Lüthy, R., \& Bowie, J. U. (1997). VERIFY3D: Assessment of protein models with three-dimensional profiles. Methods in Enzymology, 277, 396-404. doi:10.1016/S0076-6879(97)77022-8

Emini, E. A., Hughes, J. V., Perlow, D., \& Boger, J. (1985). Induction of hepatitis $A$ virus-neutralizing antibody by a virus-specific synthetic peptide. Journal of Virology, 55(3), 836-839.

Fattahian, Y., Riahi-Madvar, A., Mirzaee, R., Asadikaram, G., \& Rahbar, M. R. (2017). In silico locating the immune-reactive segments of Lepidium draba peroxidase and designing a less immune-reactive enzyme derivative. Computational Biology and Chemistry, 70, 21-30. doi:10.1016/j.compbiolchem.2017.07.003

Fonfria, E., Elliott, M., Beard, M., Chaddock, J., \& Krupp, J. (2018). Engineering botulinum toxins to improve and expand targeting and SNARE cleavage activity. Toxins, 10(7), 278. doi:10.3390/ toxins 10070278

Frevert, J. (2015). Pharmaceutical, biological, and clinical properties of botulinum neurotoxin type A products. Drugs in $R \& D, 15(1), 1-9$. doi: 10.1007/s40268-014-0077-1

Gasteiger, E., Hoogland, C., Gattiker, A., Wilkins, M. R., Appel, R. D., \& Bairoch, A. (2005). Protein identification and analysis tools on the ExPASy server. In: Walker, J. M. (Ed.), The proteomics protocols handbook (pp. 571-607). New York, NY: Humana press. doi:10.1385/159259-890-0:571

Hajighahramani, N., Nezafat, N., Eslami, M., Negahdaripour, M. Rahmatabadi, S. S., \& Ghasemi, Y. (2017). Immunoinformatics analysis and in silico designing of a novel multi-epitope peptide vaccine against Staphylococcus aureus. Infection, Genetics and Evolution, 48, 83-94. DOI: 10.1016/j.meegid.2016.12.010 doi:10.1016/ j.meegid.2016.12.010

Henriques, J., Cragnell, C., \& Skepö, M. (2015). Molecular dynamics simulations of intrinsically disordered proteins: Force field evaluation and comparison with experiment. Journal of Chemical Theory and Computation, 11(7), 3420-3431. doi:10.1021/ct501178z 
Hill, K. K., Xie, G., Foley, B. T., \& Smith, T. J. (2015). Genetic diversity within the botulinum neurotoxin-producing bacteria and their neurotoxins. Toxicon, 107, 2-8. doi:10.1016/j.toxicon.2015.09.011

Hospital, A., Andrio, P., Fenollosa, C., Cicin-Sain, D., Orozco, M., \& Gelpí, J. L. (2012). MDWeb and MDMoby: An integrated web-based platform for molecular dynamics simulations. Bioinformatics, 28(9), 1278-1279. doi:10.1093/bioinformatics/bts139

Ikai, A. (1980). Thermostability and aliphatic index of globular proteins The Journal of Biochemistry, 88(6), 1895-1898. doi:10.1093/ oxfordjournals.jbchem.a133168

Jespersen, M. C., Peters, B., Nielsen, M., \& Marcatili, P. (2017). BepiPred2.0: Improving sequence-based B-cell epitope prediction using conformational epitopes. Nucleic Acids Research, 45(W1), W24-W29. doi: $10.1093 /$ nar/gkx346

Kalyanaraman, N. (2018). In silico prediction of potential vaccine candidates on capsid protein of human bocavirus 1. Molecular Immunology, 93, 193-205. doi:10.1016/j.molimm.2017.11.024

Kamaraj, B., \& Purohit, R. (2013). In silico screening and molecular dynamics simulation of disease-associated nsSNP in TYRP1 gene and its structural consequences in OCA3. BioMed Research International, 2013, 1 (article ID 697051). doi:10.1155/2013/697051

Kelley, L. A., Mezulis, S., Yates, C. M., Wass, M. N., \& Sternberg, M. J. (2015). The Phyre2 web portal for protein modeling, prediction and analysis. Nature Protocols, 10(6), 845-858. doi:10.1038/nprot.2015.053

Kolaskar, A. S., \& Tongaonkar, P. C. (1990). A semi-empirical method for prediction of antigenic determinants on protein antigens. FEBS Letters, 276(1-2), 172-174. doi:10.1016/0014-5793(90)80535-Q

Kringelum, J. V., Lundegaard, C., Lund, O., \& Nielsen, M. (2012). Reliable B cell epitope predictions: Impacts of method development and improved benchmarking. PLoS Computational Biology, 8(12), e1002829. doi:10.1371/journal.pcbi.1002829

Lacy, D. B., Tepp, W., Cohen, A. C., DasGupta, B. R., \& Stevens, R. C. (1998). Crystal structure of botulinum neurotoxin type A and implications for toxicity. Nature Structural Biology, 5(10), 898-902. DOI: 10.1038/2338 doi:10.1038/2338

Larsen, J. E. P., Lund, O., \& Nielsen, M. (2006). Improved method for predicting linear B-cell epitopes. Immunome Research, 2(1), 2-7. doi: 10.1186/1745-7580-2-2

Lewitt, P. A., \& Trosch, R. M. (1997). Idiosyncratic adverse reactions to intramuscular botulinum toxin type A injection. Movement Disorders, 12(6), 1064-1067. doi:10.1002/mds.870120637

Li, M., Goldberger, B. A., \& Hopkins, C. (2005). Fatal case of BOTOX ${ }^{\circledR}$ related anaphylaxis? Journal of Forensic Sciences, 50(1), 169-172. doi: $10.1520 / J F S 2004196$

Lüthy, R., Bowie, J. U., \& Eisenberg, D. (1992). Assessment of protein models with three-dimensional profiles. Nature, 356(6364), 83. doi: $10.1038 / 356083 a 0$

Luvisetto, S., Gazerani, P., Cianchetti, C., \& Pavone, F. (2015). Botulinum toxin type $A$ as a therapeutic agent against headache and related disorders. Toxins, 7(9), 3818-3844. doi:10.3390/toxins7093818

Moon, I. J., Chang, S. E., \& Kim, S. D. (2017). First case of anaphylaxis after botulinum toxin type $\mathrm{A}$ injection. Clinical and Experimenta Dermatology, 42(7), 760-762. doi:10.1111/ced.13108

Negahdaripour, M., Eslami, M., Nezafat, N., Hajighahramani, N., Ghoshoon, M. B., Shoolian, E., ... Ghasemi, Y. (2017). A novel HPV prophylactic peptide vaccine, designed by immunoinformatics and structural vaccinology approaches. Infection, Genetics and Evolution, 54, 402-416. doi:10.1016/j.meegid.2017.08.002

Nezafat, N., Karimi, Z., Eslami, M., Mohkam, M., Zandian, S., \& Ghasemi, Y. (2016). Designing an efficient multi-epitope peptide vaccine against Vibrio cholerae via combined immunoinformatics and protein interaction-based approaches. Computational Biology and Chemistry, 62, 82-95. doi:10.1016/j.compbiolchem.2016.04.006

Pahil, S., Taneja, N., Ansari, H. R., \& Raghava, G. P. S. (2017). In silico analysis to identify vaccine candidates common to multiple serotypes of Shigella and evaluation of their immunogenicity. PloS One, 12(8), e0180505. doi:10.1371/journal.pone.0180505
Potocnakova, L., Bhide, M., \& Pulzova, L. B. (2016). An introduction to Bcell epitope mapping and in silico epitope prediction. Journal of Immunology Research, 2016(6760830), 1-11. doi:10.1155/2016/6760830

Rajendran, V., \& Sethumadhavan, R. (2014). Drug resistance mechanism of PncA in Mycobacterium tuberculosis. Journal of Biomolecular Structure and Dynamics, 32(2), 209-221. doi:10.1080/ 07391102.2012 .759885

Rigoni, M., Caccin, P., Johnson, E. A., Montecucco, C., \& Rossetto, O. (2001). Site-directed mutagenesis identifies active-site residues of the light chain of botulinum neurotoxin type A. Biochemical and Biophysical Research Communications, 288(5), 1231-1237. doi:10.1006/ bbrc.2001.5911

Rosenfield, L. K., Kardassakis, D. G., Tsia, K. A., \& Stayner, G. (2014). The first case report of a systemic allergy to OnabotulinumtoxinA (Botox) in a healthy patient. Aesthetic Surgery Journal, 34(5), 766-768. doi: $10.1177 / 1090820 \times 14532648$

Saha, S., \& Raghava, G. P. S. (2006). Prediction of continuous B-cell epitopes in an antigen using recurrent neural network. Proteins: Structure, Function, and Bioinformatics, 65(1), 40-48. doi:10.1002/prot.21078

Schellekens, H. (2002). Immunogenicity of therapeutic proteins: Clinica implications and future prospects. Clinical Therapeutics, 24(11), 1720-1740. doi:10.1016/S0149-2918(02)80075-3

Scott, A. B. (1981). Botulinum toxin injection of eye muscles to correct strabismus. Transactions of the American Ophthalmological Society, 79, 734-770.

Sefid, F., Rasooli, I., Jahangiri, A., \& Bazmara, H. (2015). Functional exposed amino acids of BauA as potential immunogen against Acinetobacter baumannii. Acta Biotheoretica, 63(2), 129-149. doi 10.1007/s10441-015-9251-2

Shi, J., Zhang, J., Li, S., Sun, J., Teng, Y., Wu, M., ... Hu, Y. (2015). Epitope-based vaccine target screening against highly pathogenic MERS-CoV: An in silico approach applied to emerging infectious diseases. PloS One, 10(12), e0144475. doi:10.1371/journal.pone.0144475

Silvaggi, N. R., Boldt, G. E., Hixon, M. S., Kennedy, J. P., Tzipori, S., Janda, K. D., \& Allen, K. N. (2007). Structures of Clostridium botulinum neurotoxin serotype A light chain complexed with small-molecule inhibitors highlight active-site flexibility. Chemistry \& Biology, 14(5), 533-542. doi:10.1016/j.chembiol.2007.03.014

Singh, H., Ansari, H. R., \& Raghava, G. P. (2013). Improved method for linear B-cell epitope prediction using antigen's primary sequence. PloS One, 8(5), e62216. doi:10.1371/journal.pone.0062216

Swearingen, K. E., Lindner, S. E., Shi, L., Shears, M. J., Harupa, A., Hopp, C. S., ... Sinnis, P. (2016). Interrogating the Plasmodium sporozoite surface: Identification of surface-exposed proteins and demonstration of glycosylation on CSP and TRAP by mass spectrometry-based proteomics. PLoS Pathogens, 12(4), e1005606. doi:10.1371/ journal.ppat.1005606

Wiederstein, M., \& Sippl, M. J. (2007). ProSA-web: Interactive web service for the recognition of errors in three-dimensional structures of proteins. Nucleic Acids Research, 35(Web Server), W407-W410. doi: 10.1093/nar/gkm290

Yao, B., Zhang, L., Liang, S., \& Zhang, C. (2012). SVMTriP: A method to predict antigenic epitopes using support vector machine to integrate tri-peptide similarity and propensity. PloS One, 7(9), e45152. doi: 10.1371/journal.pone.0045152

Yasmin, T., Akter, S., Debnath, M., Ebihara, A., Nakagawa, T., \& Nabi, A. N (2016). In silico proposition to predict cluster of B-and T-cell epitopes for the usefulness of vaccine design from invasive, virulent and membrane associated proteins of C. jejuni. In Silico Pharmacology, 4(5), 1-10. doi:10.1186/s40203-016-0020-y

Zarei, M., Nezafat, N., Rahbar, M. R., Negahdaripour, M., Sabetian, S. Morowvat, M. H., \& Ghasemi, Y. (2018). Decreasing the immunogenicity of arginine deiminase enzyme via structure-based computational analysis. Journal of Biomolecular Structure and Dynamics, 1-14 [published online]. doi:10.1080/07391102.2018.1431151 


\section{in_silico_mutagenesis.pdf by Sulistyo Vianney}

Submission date: 01-Feb-2021 02:07PM (UTC+0700)

Submission ID: 1498884848

File name: in_silico_mutagenesis.pdf (2.51M)

Word count: 8205

Character count: 43653 


\section{In silico mutagenesis: decreasing the immunogenicity of botulinum toxin type $A$}

\section{Stanley Evander Emeltan Tjoa, Yoanes Maria Vianney \& Sulistyo Emantoko} Dwi Putra

To cite this article: Stanley Evander Emeltan Tjoa, Yoanes Maria Vianney \& Sulistyo Emantoko Dwi Putra (2019) In silico mutagenesis: decreasing the immunogenicity of botulinum toxin type A, Journal of Biomolecular Structure and Dynamics, 37:18, 4767-4778, DOI: 10.1080/07391102.2018.1559100

To link to this article: https://doi.org/10.1080/07391102.2018.1559100

View supplementary material ¿

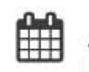

Accepted author version posted online: 17

Dec 2018.

Published online: 16 Jan 2019.

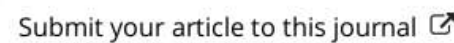

Џ Article views: 97

a

View related articles \lceil

View Crossmark data $₫$ 


\title{
In silico mutagenesis: decreasing the immunogenicity of botulinum toxin type A
}

\author{
Stanley Evander Emeltan Tjoa*, Yoanes Maria Vianney* and Sulistyo Emantoko Dwi Putra \\ Faculty of Biotechnology, University of Surabaya, Surabaya, East Java, Indonesia \\ Communicated by Ramaswamy H. Sarma
}

\begin{abstract}
Botulinum toxin serotype $A$ is a prominent therapeutic enzyme, for both clinical and cosmetic uses. Since this protein is produced by bacteria, it exhibits an allergenic effect when subjected to human therapy. Protein mutagenesis is one method to improve the characteristics of protein. However, in silico study is needed to give suggestion of which amino acid should be mutated. Hence, a lot of money and time can be saved. This study initially screened which residue of the Botulinum toxin serotype A is B-cell epitopes both linearly and conformationally. By overlapping the B-cell epitopes with the excluded conserve sequence, seven residues were allowed to be mutated. There were two proposed muteins showing a reduction in the antigenicity probability: $\triangle E 147, E 510 \mathrm{~F}, \mathrm{~T} 1062 \mathrm{~F}, \Delta E 1080, \mathrm{~N} 1089 \mathrm{M}$ and $\triangle Q 1090$; and $\triangle E 147, E 510 F, T 1062 F, E 1080 W, N 1089 M$ and $\triangle Q 1090$. Molecular dynamics simulation of the 3D proposed muteins indicated an increase of flexibility in both muteins compared to that in the native protein. Both muteins have lower antigenicity. In addition, they are similar in structure, stability and functionality compared to the native protein.
\end{abstract}

Abbreviations: 3D: three-dimensional; BoNT: botulinum neurotoxins; FNN: feed-forward neural network; GRAVY: grand average of hydropathy; MSMS: Michel Sanner's molecular surface; MD simulation: molecular dynamics simulation; NPT: constant number, pressure and temperature; NVT: constant number, volume and temperature; SASA: solvent-accessible surface area; $R_{\mathrm{g}}$ : radius of gyration; RMSD: rootmean-square deviation; RMSF: root-mean-square fluctuation; SVM: support vector machine; US FDA: United States Food and Drug Administration
ARTICLE HISTORY

Received 7 August 2018

Accepted 9 December 2018

KEYWORDS

Bioinformatics; botulinum toxin type A; B-cell epitopes prediction; immunogenicity: in silico mutagenesis

\section{Introduction}

Botulinum neurotoxins (BoNT) are potent toxins which are produced mainly by Clostridium botulinum, and the other producers are $C$. argentinense, $C$. baratii and $C$. butyricum. Eight serotypes of BoNT (A-H) have been characterized. These toxins are zinc metalloprotease that consists of heavy chain, light chain and translocation domain (Hill, Xie, Foley, \& Smith, 2015). Botulinum toxin was first known to cause food poisoning resulting in muscle paralysis. Botulinum toxin affected muscle neuron after its internalization of the botox to the cytosol by the heavy chain via SV2 receptor protein; the light chain of toxin cut the SNAP-25 (by BoNT/A, $/ E$, and/ C), VAMP/synaptobrevin (by BoNT/B,/D,/F, G) and syntaxin (BoNT/C). These proteins are well known as SNARE complex components which act as a docking protein for neurotransmitter exocytosis (Carruthers \& Carruthers, 2005; Luvisetto, Gazerani, Cianchetti, \& Pavone, 2015).

Among those toxin serotypes, BoNT/A is the most utilized protein as a therapeutic agent for muscle relaxation, since botox is the one to be well characterized, tested and approved by the US Food and Drug Administration (FDA)
(Brooks, 1954; Frevert, 2015; Scott, 1981). Since then, BoNT/A has been widely used for many cosmetics and physiologic disorders, such as blepharospasm, hemifacial spasm, dystonia, hyperhidrosis, migraine and neuropathic pain (neuralgia) (Babiloni, Kapos, \& Nixdorf, 2016; Bentivoglio, Del Grande, Petracca, Lalongo, \& Ricciardi, 2015; Carruthers \& Carruthers, 2005; Castelao et al., 2017). BoNT/A as a therapeutic protein is also stimulated by biotechnology revolution. This encourages the market for more innovation and its unique properties dedicated to increase the performance of the protein by many industries around the world (Dressler, 2016).

Since BoNT/A is produced originally by bacteria, this protein is considered as a foreign antigen by human immune system. Antibody secretion by B-cell can neutralize the protein and may reduce its efficacy (Schellekens, 2002). Although first doubtfully reported to induce allergic and even anaphylaxis reaction due to the possibility of other foreign substances in the preparation (LeWitt \& Trosch, 1997; Li, Goldberger, \& Hopkins, 2005), BoNT/A is proven to be able to induce immunogenic and even anaphylaxis reaction, which is life-threatening (Careta, Delgado, \& Patriota, 2015;

CONTACT Sulistyo Emantoko Dwi Putra emantoko@staff.ubaya.acid _- Faculty of Biotechnology, University of Surabaya, Kalirungkut Road, 60292 Surabaya, East Java, Indonesia.

* Both authors contributed equally. 
Moon, Chang, \& Kim, 2017; Rosenfield, Kardassakis, Tsia, \& Stayner, 2014). Indeed, BoNT/A can induce the production of antibody (IgG isotype) (Dressler \& Bigalke, 2017). With the currently vast and diverse market of BoNT/A, it is beneficial to tackle the allergenic problems as one of the different technologies proposed for its development.

Bioinformatics offer many algorithms to predict amino acid residues that induce immunogenic reaction. B-cells can detect both linear (continuous) and conformational (discontinuous) epitopes unique to human protein although most of the B-cell epitopes are conformational or fold-dependent (Kringelum, Lundegaard, Lund, \& Nielsen, 2012; Yao, Zhang, Liang, \& Zhang, 2012). Modifications of these residues can alter the antigenicity (Zarei et al., 2018). Compared to time-consuming and costly experimental approaches, a wide variety of computational tools, which are publicly available, are highly recommended to predict the B-cell epitopes to reduce or minimize cost (Kolaskar \& Tongaonkar, 1990; Kringelum et al., 2012; Potocnakova, Bhide, \& Pulzova, 2016; Saha \& Raghava, 2006; Singh, Ansari, \& Raghava, 2013; Yao et al., 2012).

Few studies to mutate BoNT/A to enhance the botox performance have been attempted (Fonfria, Elliott, Beard, Chaddock, \& Krupp, 2018). This paper focuses on the strategy of BoNT/A rational design to reduce the immunogenicity of the protein by mutating the predicted B-cell epitopes. B-cell epitopes were predicted both conformationally and linearly; these epitopes overlapped with the conserved region predicted by the thermodynamic analysis and the essential site of the toxin for the activity (Rigoni, Caccin, Johnson, Montecucco, \& Rossetto, 2001). Then, the new protein was also modelled and evaluated for its physical properties and stability.

\section{Materials and methods}

In general, the steps used in this methodology were (1) determination of B-cell linear and conformational epitopes from BoNT/A, (2) determination of conserved region and (3) mutagenesis of BoNT/A and evaluation of antigenic, physical properties and stability.

\subsection{Sequence retrieval}

The protein used in this paper was botulinum toxin serotype A (BoNT/A) (PDB ID: 3BTA) (Lacy, Tepp, Cohen, DasGupta, \& Stevens, 1998). Protein domain and features were obtained from UniProt (https://www.uniprot.org/uniprot/P10845). Three amino acid residues, 262, 266 and 366, are important for the enzymatic activity. Thus, they should not be mutated (Rigoni et al., 2001). The sequence was saved in the Fasta format, and the new 3D structure of the protein from the RCSB PDB was stored for further studies.

\subsection{Immuno-informatic analyses}

\subsubsection{Linear B-cell epitopes prediction}

Several online tools were used to predict the linear B-cell epitopes. BepiPred-2.0 (http://www.cbs.dtu.dk/services/
BepiPred/) (Jespersen, Peters, Nielsen, \& Marcatili, 2017) was used to predict epitopes based on random forest algorithm trained on epitopes amino acids from the crystal structure of protein. Threshold of 0.5 and sensitivity of 0.6 were employed. Additionally, Bepipred server, accessed from IEDB (http://tools.immuneepitope.org/bcell/), was used to predict the linear B-cell epitopes by using hidden Markov model and propensity scale method (Larsen, Lund, \& Nielsen, 2006). The threshold of 0.35 with the sensitivity of 0.49 and the specificity of 0.75 was employed.

SVMTriP (http://sysbio.unl.edu/SVMTriP/) (Yao et al., 2012) was used to predict linear B-cell epitopes that integrate Tripeptide similarity and propensity score utilizing support vector machine (SVM) algorithm. Another tool was used to predict the linear epitopes of B-cell (http://imed.med.ucm.es/ Tools/antigenic.html) and to evaluate the occurrences of amino acids that acted as epitope in protein experimentally (Kolaskar \& Tongaonkar, 1990). Briefly, the average propensity of central amino acid residue of every 7-mer was evaluated and compared with the average propensity of the whole protein. The threshold to consider an antigenic amino acid residue was 1.0 . Then, every 8 -mer where all residues were above the threshold is considered as an epitope. ABCpred (http://crdd.osdd.net/raghava/abcpred/) was employed to predict continuous B-cell epitopes based on machine learning techniques, such as feed-forward (FNN) and recurrent neural network (RNN). As many as 20 mers could be predicted as epitope (Saha \& Raghava, 2006). The score used for the threshold was 0.81 .

\subsubsection{Conformational B-cell epitopes prediction}

Discotope 2.0 (http://www.cbs.dtu.dk/services/DiscoTope/) was used to predict conformational B-cell epitopes from the calculation of surface accessibility and novel propensity amino acid score (Kringelum et al., 2012). A threshold of -1 with $30 \%$ sensitivity and $85 \%$ specificity was used. CBTOPE (Conformational B-cell Epitope Prediction) (http://crdd.osdd. net/raghava/cbtope/) server was also employed with the accuracy of about $84 \%$. This tool used SVM algorithm to train the program with the amino acid composition and physicochemical profile to predict the epitopes (Ansari \& Raghava, 2010). Threshold of -0.3 was used, and residues with the scale above 5 were considered epitopes. Moreover, exposed surface was analyzed with Emini surface accessibility which was accessed at IEDB Analysis Resource (Emini, Hughes, Perlow, \& Boger, 1985). Score of surface probability that resulted above one was predicted to be accessible on the surface of the protein.

\subsubsection{Examination of the conserved region}

Entropy method provided by Swiss Model ExPASy was used to determine the conserved region of the protein (Arnold, Bordoli, Kopp, \& Schwede, 2006; Benkert, Biasini, \& Schwede, 2011; Biasini et al., 2014). Entropy score below 2 is considered conserved residue which will be excluded from mutagenesis analysis. 

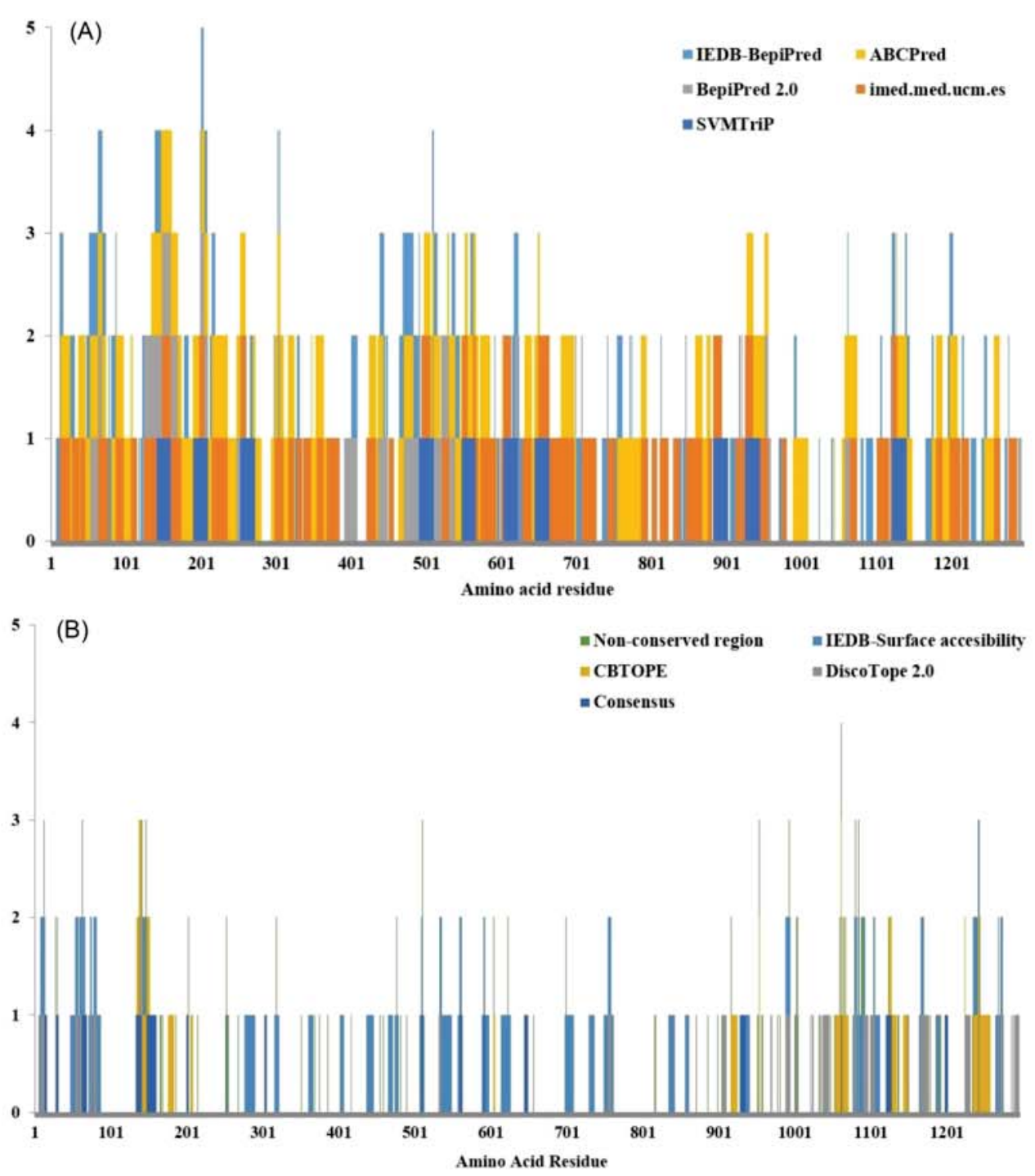

Figure 1. Graphical illustration of linear B-cell epitopes (A) and overlap of consensus from the linear B-cell epitopes with the conformational B-cell epitopes and non-conserved region $(B)$ to determine which residues are allowed to mutate.

\subsubsection{In silico mutagenesis of the predicted residues}

In silico mutagenesis and stability examination of the mutein were done using I-Mutant 2.0 (http://folding.biofold.org/imutant/i-mutant2.0.html) for every single substitution mutation of the determined residues (Capriotti, Fariselli, \& Casadio, 2005). The condition for the mutation was $\mathrm{pH} 7.4$ at the temperature of $37^{\circ} \mathrm{C}$, which conforms to human physiological condition. Mutation was allowed if the difference of the Gibbs free energy was positive which indicated an increase of protein stability. The difference of the Gibbs free energy was measured with the formula of $\Delta G$ (mutein) $\Delta G$ (wild-type protein). The residue that did not contain any positive value of difference of the Gibbs free energy was tried for deletion-type mutation. The proteins with mutated residues were subjected to the B-cell epitopic probability with VaxiJen (http://www.ddg-pharmfac.net/vaxijen/VaxiJen/
VaxiJen.html). This tool used auto cross-covariance pre-processing of protein while detecting antigenicity of residues with the help of $z$-descriptors as physicochemical parameters (Doytchinova \& Flower, 2007).

\subsection{Immuno-informatic analyses}

2.3.1. 3D Modelling of mutated protein and validation 3D protein was predicted and evaluated by PHYRE2 Protein Fold Recognition Server (http://www.sbg.bio.ic.ac.uk/phyre2). This tool displays the accuracy comparable to the widely used web servers for protein modelling, such as I-TASSER or Swiss-Model, but it is easier to use. PHYRE2 also provided investigator tools for 3D model evaluation and validation (Kelley, Mezulis, Yates, Wass, \& Sternberg, 2015). The 
Table 1. Analysis of substitution mutation stability with difference in Gibbs' free energy method by I-Mutant 2.0 Condition of mutation was set at $\mathrm{pH}$ of 7.4 and temperature of $37^{\circ} \mathrm{C}$

\begin{tabular}{|c|c|c|c|c|c|c|c|}
\hline$\Delta \Delta G$ & E147 & E510 & T1062 & E1080 & D1088 & N1089 & Q1090 \\
\hline V & -0.98 & -0.54 & -0.47 & 0.73 & -1.13 & -1.23 & -1.50 \\
\hline L & -1.18 & -0.50 & -0.88 & 0.98 & -1.25 & -0.62 & -1.21 \\
\hline 1 & -0.71 & -0.04 & -0.12 & 1.31 & -0.81 & -0.04 & -1.24 \\
\hline M & -0.69 & -0.01 & 0 & 1.01 & -0.74 & 0.01 & -1.76 \\
\hline $\mathrm{F}$ & -0.20 & 0.86 & 0.29 & 2 & -0.13 & -0.17 & -0.71 \\
\hline W & -0.89 & -0.07 & -0.03 & 1.06 & -1.21 & -0.87 & -1.33 \\
\hline Y & -1.60 & -0.28 & 0.47 & 1.25 & -0.47 & -0.63 & -1.52 \\
\hline G & -2.05 & -1.15 & -1.20 & 0.13 & -1.51 & -0.91 & -1.92 \\
\hline A & -2.12 & -1.17 & -0.54 & -0.13 & -2 & -1.61 & -2.78 \\
\hline P & -2.48 & -2.14 & -1.31 & -0.96 & -2.50 & -2.05 & -2.87 \\
\hline$S$ & -1.74 & -0.66 & -0.09 & 0.06 & -1.06 & -1.12 & -2.03 \\
\hline $\mathrm{T}$ & -1.29 & -0.18 & & 0.81 & -1.2 & -0.86 & -1.58 \\
\hline C & -0.65 & -0.29 & -0.46 & 0.3 & -0.77 & -0.45 & -1.59 \\
\hline $\mathrm{H}$ & -2.15 & -1.19 & -1.42 & -0.48 & -0.74 & -1.46 & -2.31 \\
\hline$R$ & -1.69 & -0.25 & 0.65 & 0.68 & -1.23 & -0.44 & -2.01 \\
\hline $\mathrm{K}$ & -2.37 & -1.42 & -0.30 & -0.55 & -1.39 & -2.07 & -2.66 \\
\hline Q & -1.78 & -0.94 & -0.69 & 0.26 & -1.12 & -1.07 & \\
\hline $\mathrm{E}$ & & & 0.11 & & -1.06 & -0.68 & -1.84 \\
\hline $\mathrm{N}$ & -2.62 & -1.24 & -0.31 & 0.26 & -1.45 & & -3.11 \\
\hline D & -1.75 & -0.58 & 0.33 & 0.49 & & -0.89 & -1.74 \\
\hline
\end{tabular}

The bold values indicated the positive values of $\Delta \Delta G$

evaluations of 3D protein quality provided by PHYRE2 were ProQ2 quality assessment, HHsearch alignment confidence, analysis of clashes, rotamers and disorder analysis based on Disopred. ProtParam was also used to analyze all protein properties and stability (Gasteiger et al., 2005). Ramachandran plot, however, was analyzed by RAMPAGE (http://mordred.bioc.cam.ac.uk/ rapper/rampage.php) ProSAweb (https://prosa.services.came.sbg.ac.at/prosa.php) was employed to evaluate the $3 D$ protein quality in terms of $Z$ score and residue energies plot. A good model is indicated with a $Z$-score inside the range characteristics of scores from other native proteins with similar sizes and groups, while the energy plot shows the local model quality for each central residue in every 40-mer fragment (Wiederstein \& Sippl, 2007). In addition, the protein model quality was also analyzed with VERIFY3D (Lüthy, Bowie, \& Eisenberg, 1992; Bowie, Lüthy, \& Eisenberg, 1991) and ERRAT (Colovos \& Yeates, 1993) from SAVES 5.0 web servers (http://servicesn.mbi.ucla. edu/SAVES/).

\subsubsection{Molecular dynamics (MD) simulation}

The 3D models of mutein generated and validated from PHYRE2 were subjected to MD simulation at MDweb (http:// mmb.irbbarcelona.org/MDWeb/) (Hospital et al., 2012). Gromacs Full MD setup with Gromos $53 a 6$ force field was employed. The system was solvated with TIP3P water molecules with a spacing distance of $15 \AA$ around the protein. $\mathrm{Na}$ + and $\mathrm{Cl}$ - were added to neutralize the system until the concentration reached $50 \mathrm{mM}$. Initially, heavy atoms were restrained with a force constant of $500 \mathrm{KJ} / \mathrm{mol} \times \mathrm{nm}^{2}$. The system was further equilibrated under NPT and NVT ensembles. The solvent was heated to $300 \mathrm{~K}$, and the force for restraining the heavy atoms was gradually diminished until the simulation was done without restraint. The temperature and pressure were kept at default with velocity-rescale and Parinello-Rahman algorithms, respectively.
The root-mean-square deviation (RMSD) aligned through trajectory, root-mean-square fluctuation (RMSF) of all residues, the radius of gyration $(\mathrm{Rg})$, solvent-accessible surface area (SASA) and secondary structure projection aligned through trajectory for each protein were computed. SASA was measured with StrucTools (https://hpcwebapps.cit.nih. gov/structbio/basic.html) with an MSMS program (Michel Sanner's Molecular Surface). The surface probe size was 1.4 $\AA$, and the surface of only atom in general 20 amino acids was calculated. The same tool was used to calculate which residues of the protein contributed to the following secondary structure: $\alpha$-helix, $\beta$-strand, $\beta$-turn, $\beta$-bridge, random coil and 310-helix. The secondary structure projection plot was built by calculating the amount of residue that composed each secondary structure types within the simulation time. Intramolecular hydrogen bonds were analyzed by first removing all water molecules and ions from the trajectory. Hydrogen bond calculation tool (http://cib.cf.ocha.ac.jp/ bitool/HBOND/) was employed to calculate the amount of hydrogen bond from each pdb file. The MD was done in 26 snapshots in which each snapshot was $0.5 \mathrm{~ns}$. The total time for MD simulation was 13,000 ps.

\section{Results and discussion}

BoNT/A market is globally diverse at the moment, with one industry producing its own formulation and protein modification for its novelty (Dressler, 2016). Therefore, it is interesting to develop the low antigenicity type of this protein since this protein is a foreign one. In silico method provides an initial study for mutagenesis to suggest which amino acid residue should be mutated to show lower antigenicity while still retaining its stability and activity compared to the native protein.

\subsection{B-cell epitopes prediction}

Various tools are available in a lot of web-server software to predict antigenicity of a protein, with regard to the previously known 3D structure of the BoNT/A. BoNT/A has been characterized with $\mathrm{X}$-ray crystallography with multiple isomorphous replacement as phasing approach (Lacy et al., 1998). The downloaded PDB files (3BTA) were directly used for the determination of B-cell epitopes. The study, thus, can be directed to the determination of the B-cell epitopes.

Some researchers used web-server tools to evaluate the antigenicity of protein residues (Fattahian, Riahi-Madvar, Mirzaee, Asadikaram \& Rahbar, 2017; Zarei et al., 2018). In total, five tools were employed to predict the linear B-cell conformation, and three types of web-server software were used to predict the conformational B-cell epitopes with various amino acid lengths. The residues obtained from the linear B-cell epitopes prediction must comply with at least three of the five tools. The consensus residues obtained using this method were overlaid with the result of the prediction of the conformational B-cell epitopes and the nonconserve region to select the epitopes which are permitted to be mutated. The selected epitopes can be seen 
Table 2. Deletion of the determined residues with no increase of $\Delta \Delta G$ value.

\begin{tabular}{lllllll}
\hline & $\Delta E 147$ & $\Delta D 1088$ & $\Delta Q 1090$ & $\Delta E 147 \Delta D 1088$ & $\Delta E 147 \Delta Q 1090$ & $\Delta E 147 \Delta D 1088 \Delta Q 1090$ \\
\hline VaxiJen Score & 0.3929 & 0.3915 & 0.3906 & 0.391 & 0.3901 & 0.3903 \\
\hline
\end{tabular}

The initial VaxiJen score of the BoNT/A is 0.3934 .

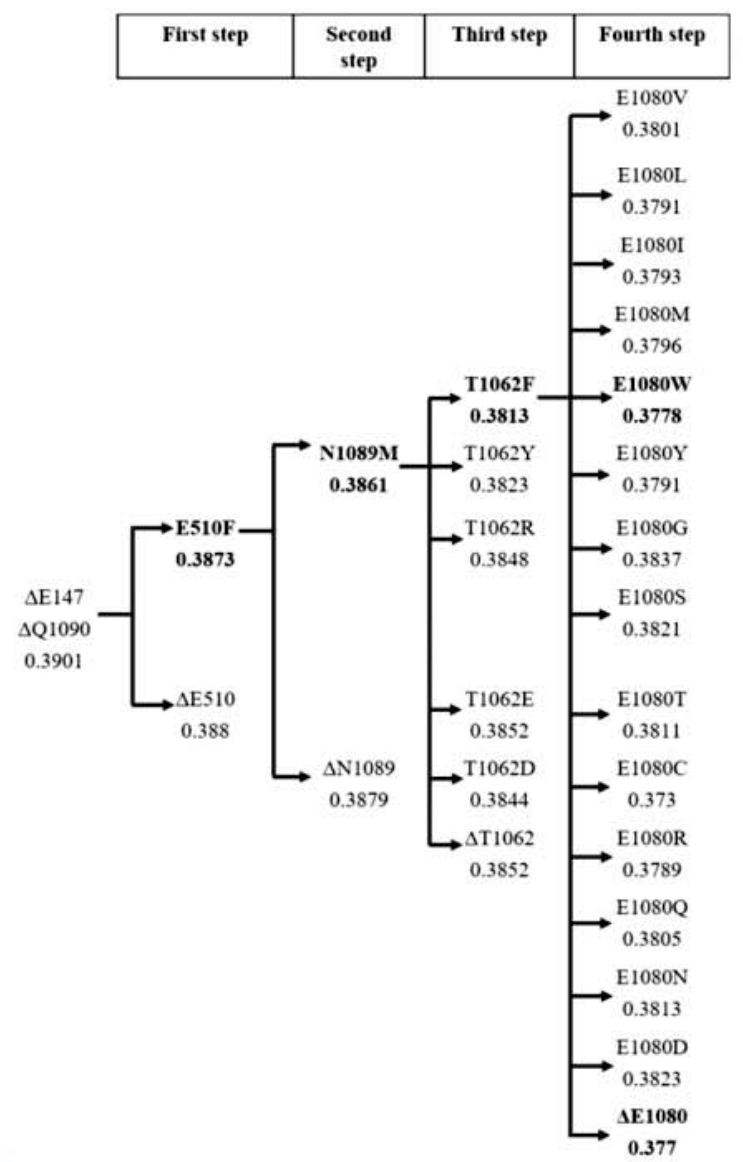

Figure 2. Sequential substitution in silico mutagenesis strategy flowchart with the help of the reduction of immunogenicity score from VaxiJen. The mutation was retained after each step, while the initial protein used the result of the deletion mutation indicated in bold.

schematically in Figure 1. The same tools can also be used to predict the antigenicity of the protein directed to vaccine design of emerging pathogens (Bazmara et al., 2017; Hajighahramani et al., 2017; Kalyanaraman, 2018; Negahdaripour et al., 2017; Nezafat et al., 2016; Pahil, Taneja, Ansari, \& Raghava, 2017; Shi et al., 2015; Yasmin et al., 2016).

Conserved region determined by entropy method (Supplementary data 1) usually contributed to the structural and functional properties. This region was, therefore, excluded in order to ensure the stability and the conformation of the muteins (Zarei et al., 2018). Based on the linear B-cell epitopes, conformational B-cell epitopes, surface accessibility and conserved region prediction, seven residues were obtained out of 1296 amino acids residue. T1062 was the overlapping region with consensus linear epitopes and two other conformational residues, whereas E147, E510, E1080,
D1088, N1089 and Q1090 were obtained from the overlapping process of consensus linear epitopes and one of the Bcell conformational epitopes prediction tools. Indeed, most polar amino acids were predicted as B-cell epitopes since these amino acids tend to be at the surface of a protein that can be recognized by immune system cells and antibody (Swearingen et al., 2016). While referring to DiscoTope 2.0 conformational B-cell prediction, many of the residues were located near the $\mathrm{N}$-terminal $(8-141)$ and $\mathrm{C}$-terminal (906-1296) which were normally exposed (Sefid, Rasooli, Jahangiri, \& Bazmara, 2015).

\subsection{In silico mutagenesis of BoNT/A and $3 D$ modelling and validation of the muteins}

1-Mutant 2.0 is able to mutate the candidate amino acid with the 19 other amino acids. Table 1 summarizes the difference in Gibbs free energy after a substitution mutation. Positive value indicated that the residue is allowed to mutate into the specified amino acid, correlating with an increase in stability after the protein has been mutated due to the positive Gibbs free energy.

In general, the strategies for the in silico mutagenesis started from the deletion analysis with the amino acids that did not contain any positive values from the result of 1 Mutant 2.0 analysis. Table 2 shows the result of the combination analysis with the help from VaxiJen for the immunogenicity score and bacteria as the source organism. Although the deletion at D1088 alone reduced the immunogenicity score, the combined score of all three deletions was still higher than when the deletions were only at E147 and Q1090. Then, mutagenesis strategy was continued with the serial mutation with the other amino acids with positive content of $\Delta \Delta G$. The VaxiJen score can be seen in Figure 2.

In summary, there were two proposed mutations. Option one was $\triangle E 147, E 510 \mathrm{~F}, \mathrm{~T} 1062 \mathrm{~F}, \triangle E 1080, \mathrm{~N} 1089 \mathrm{M}, \triangle Q 1090$ with a VaxiJen score of 0.377. Option two was $\triangle E 147$, E510F, $\mathrm{T} 1062 \mathrm{~F}, \mathrm{E} 1080 \mathrm{~W}, \mathrm{~N} 1089 \mathrm{M}, \Delta \mathrm{Q} 1090$ with a VaxiJen score of 0.3778. Both proposed muteins generated the two lowest VaxiJen scores which were analyzed from the total sequence. Changing the immunogenic residue into non-polar amino acids resulted in the less-immunogenicity protein (Zarei et al., 2018). One hotspot residue showed by IEDB with a good surface accessibility promoting mutability was residue 1079-1092. This residue was also separately analyzed with the VaxiJen. Both muteins showed lower VaxiJen score in the hotspot residue, but option two revealed a lower VaxiJen score than option one (Table 3).

These two proposed muteins were subjected to 3D protein modelling and continued with the validation of the analysis together with the native protein (Figure 3). Protein model validation that was initially analyzed by various PHYRE2 investigators revealed no major difference between 
Table 3. Immunogenic analysis by VaxiJen of the muteins, both total protein sequence and the selected hotspot residue (1079-1092).

\begin{tabular}{llll}
\hline Native & Epitope (1079-1092) & VaxiJen score of protein & VaxiJen score of surface accessibility \\
\hline Native protein & LNEKEIKDLYDNQS & 0.3934 & 0.5113 \\
First option $(\triangle E 147$, E510F, T1062F, $\triangle E 1080$, N1089M, $\triangle Q 1090)$ & LNKEIKDLYDMS & 0.377 & 0.3388 \\
Second option ( $\triangle E 147$, E510F, T1062F, E1080W, N1089M, $\Delta Q 1090)$ & LNWKEIKDLYDMS & 0.3778 & 0.1276 \\
\hline
\end{tabular}

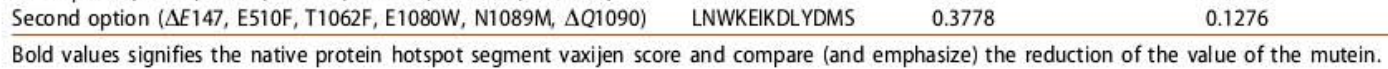
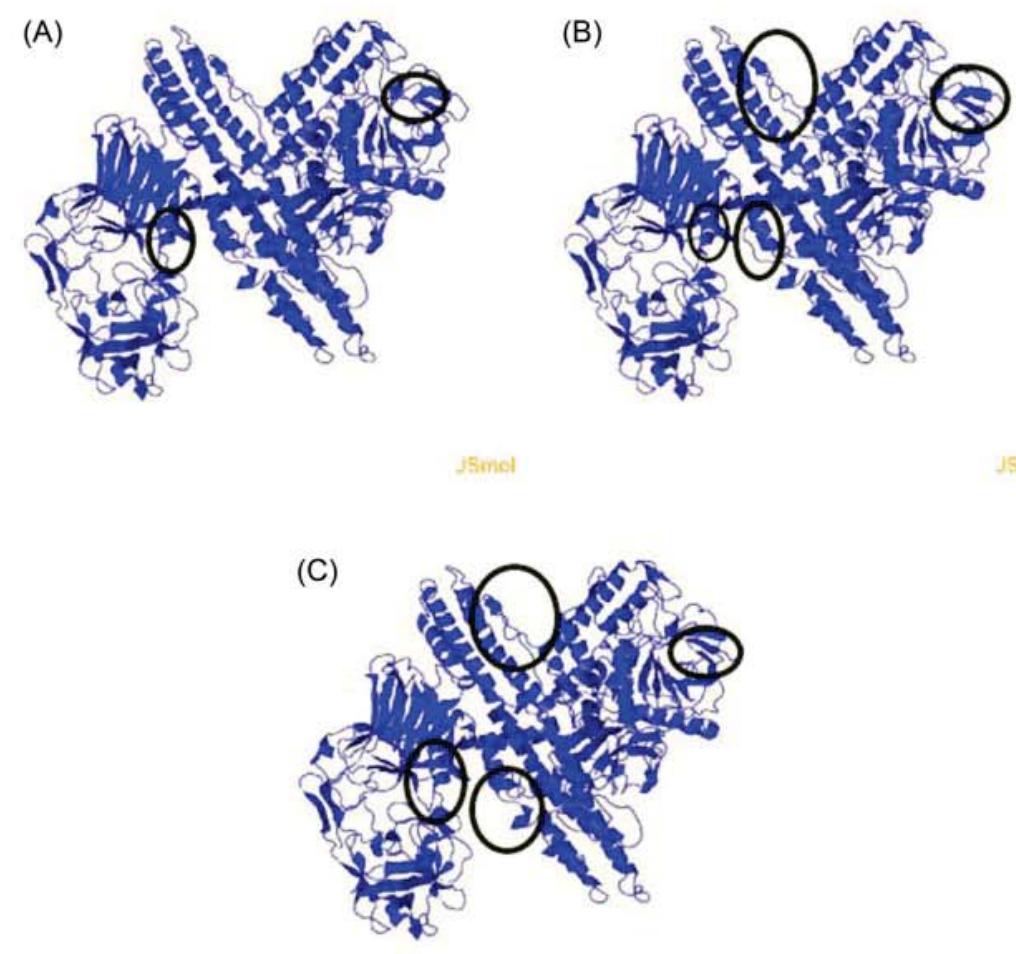

jSinal

Figure 3. 3D modelling of the first-option mutein (A), second-option mutein (B) and native BoNT/A (C) built by PHYRE2. The area indicated with circle is the one that contains difference in $3 \mathrm{D}$ structure with the native protein.

Table 4. Validation of both mutein 3D models built by PHYRE2.

\begin{tabular}{lccc}
\hline \multicolumn{1}{l}{ Table 4. Validation of both mutein 3D models built by PHYRE2. } \\
\hline & Native BoNT/A & $\begin{array}{c}\text { First-option mutein }(\Delta E 147, \text { E510F, } \\
\text { T1062F, } \Delta E 1080, N 1089 \mathrm{M}, \Delta Q 1090)\end{array}$ & $\begin{array}{c}\text { Second-option mutein }(\Delta E 147, \text { E510F, } \\
\text { T1062F, E1080W, N1089M, } \Delta Q 1090)\end{array}$ \\
\hline Ramachandran analysis: & & & $89.7 \%$ \\
Favoured region & $81.9 \%$ & $89.7 \%$ & $7.8 \%$ \\
Allowed region & $13.4 \%$ & $8 \%$ & $2.5 \%$ \\
Outlier region & $4.7 \%$ & $2.3 \%$ & -13.82 \\
Z-Score of overall model quality & -13.81 & -13.51 & $80.82 \%$ (pass) \\
Average 3D-1D score & $83.79 \%$ (pass) & $81.12 \%$ (pass) & 85.7831 \\
ERRAT overall quality factor A & 72.7273 & 85.7831 & 80.8081 \\
ERRAT overall quality factor B & 69.7789 & 80.9886 & \\
\hline
\end{tabular}

the mutein and native protein (Supplementary Data 2). Further validation analyses can be seen in Table 4 .

Both Ramachandran plot analyses of the mutein yield lower residual values for the outlier region compared to those of the native BoNT/A. The $z$-score from the PROSAweb was similar to the QMean from the SWISS-MODEL, indicating similarity of the $3 \mathrm{D}$ model to the experimental protein with similar size (Benkert, Tosatto, \& Schomburg, 2008). The $z-$ score of the first-option mutein was within the range of the other native protein with similar size, which indicated an appropriate model (Figure $4(B)$ ). Both the $z$-score of the native protein and the second-option mutein were not in the range in the plot (Figure $4(A, C)$ ). However, the $z$-score of the second-option mutein was closer to that of the native BoNT/A protein. Due to its closeness in its $z$-score to the native BoNT/A, the second-option mutein model can also be considered as valid. The local energy plots of each residue were similar to the $3 \mathrm{D}$ model protein, and these positive values of the energies could be found in the native protein as well (Figure 4(D-F)). VERIFY3D compared the 3D model with the amino acid sequence (1D) and its environment (Eisenberg, Lüthy, \& Bowie, 1997). All the 3D protein models 
(A) $2.500 m \cdot 13.81$

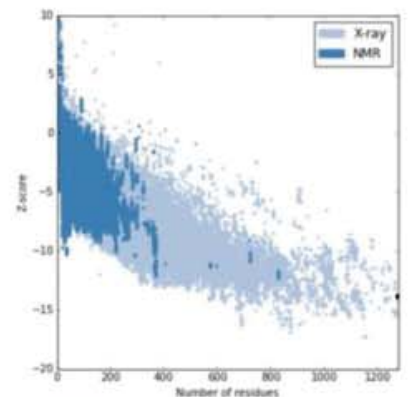

(D)

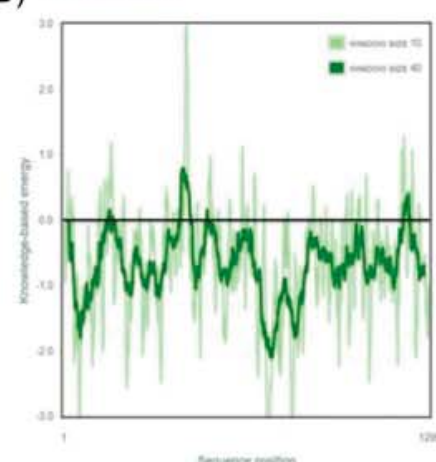

(B)

Overall model quality
25000
-1351

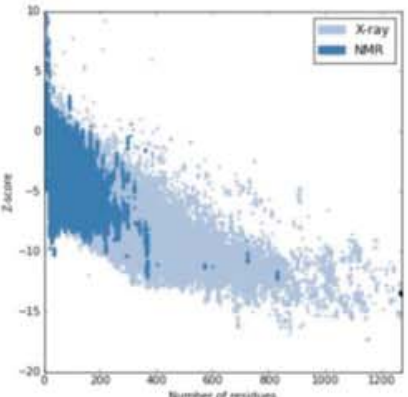

(E)
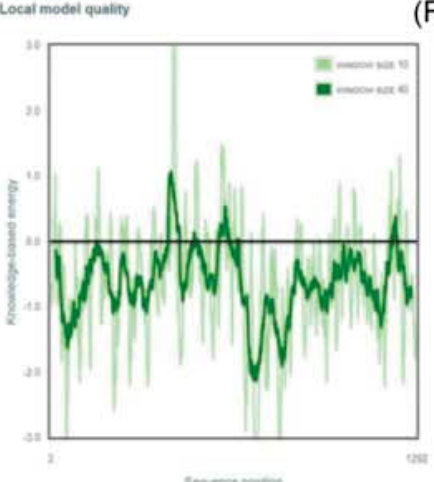

(C) Overall model quality $2.5000=-13.82$

Figure 4. 3D model validation by PROSAweb. (A-C) Overall model quality with the use of $z$-score of the native BoNT/A (A), first-option mutein (B) and secondoption mutein (C). The black dot indicated the (D-F) comparison of the residue energy plot for the local model quality for every 10 and 40 residues windows for (D) native BoNT/A, (E) first-option mutein and (F) second-option mutein.

Table 5. Native BoNT/A and both mutein properties, stability and hydrophilicity analysis.

\begin{tabular}{|c|c|c|c|}
\hline & Native BoNT/A & $\begin{array}{l}\text { First-option mutein ( } \triangle E 147, \text { E510F, } \\
\text { T1062F, } \Delta E 1080, N 1089 \mathrm{M}, \Delta Q 1090)\end{array}$ & $\begin{array}{l}\text { Second-option mutein ( } \triangle E 147, \text { E510F, } \\
\text { T1062F, E1080W, N1089M, } \Delta Q 1090)\end{array}$ \\
\hline Isoelectric point & 6.05 & 6.31 & 6.31 \\
\hline Molecular weight & 149453.89 & 149148.75 & 149334.97 \\
\hline Instability index ${ }^{a}$ & 29.35 & 29.33 & 29.05 \\
\hline Aliphatic index ${ }^{b}$ & 90.24 & 90.45 & 90.38 \\
\hline $\begin{array}{l}\text { Grand average of hydropathic- } \\
\text { ity (GRAVY) }\end{array}$ & -0.366 & -0.347 & -0.347 \\
\hline Estimated half-life ${ }^{d}$ & $>30 \mathrm{~h}$ & $>30 \mathrm{~h}$ & $>30 \mathrm{~h}$ \\
\hline
\end{tabular}

The protein is considered stable if the instability index is below 40 .

${ }^{b}$ The protein is considered stable if the aliphatic index is more positive.

cProtein is considered more hydrophobic if the value is more positive.

${ }^{d}$ The half-life of the protein was predicted using mammalian reticulocytes as model in vivo.

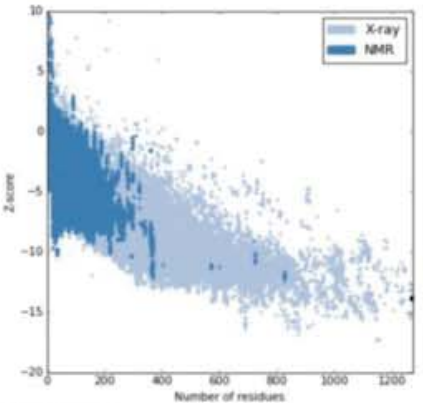

(F)

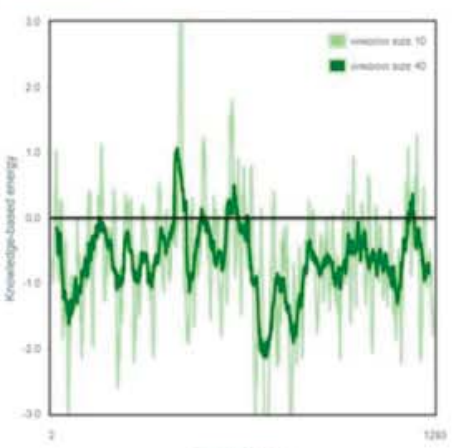

Table 6. Average values of RMSD, $R_{g}$ and RMSF for the native BoNT/A and both muteins.

\begin{tabular}{|c|c|c|c|}
\hline & Native BoNT/A & $\begin{array}{l}\text { First-option mutein ( } \triangle E 147, \text { E510F, } \\
\text { T1062F, } \Delta E 1080, N 1089 \mathrm{M}, \Delta Q 1090)\end{array}$ & $\begin{array}{l}\text { Second-option mutein }(\Delta E 147, \text { E510F, } \\
\text { T1062F, E1080W, N1089M, } \Delta Q 1090)\end{array}$ \\
\hline RMSD & $9.4649 \AA$ & $9.4564 \AA$ & $9.4552 \AA$ \\
\hline$R_{g}$ & $7.6025 \AA$ & $7.5973 \AA$ & $7.5915 \AA$ \\
\hline SASA & $501.814 \mathrm{~nm}^{2}$ & $508.462 \mathrm{~nm}^{2}$ & $502.185 \mathrm{~nm}^{2}$ \\
\hline RMSF & $0.3783 \AA$ & $0.3833 \AA$ & $0.3892 \AA$ \\
\hline
\end{tabular}

yield a result of more than $80 \%$ from its residues with a 3D-1D score $>=0.2$ (Table 4). The non-bonded interactions from the six combinations of Carbon (C), Nitrogen (N) and Oxygen $(\mathrm{O})$ and plotted the error function from every nineresidue sliding windows were analyzed using ERRAT (Colovos \& Yeates, 1993). The ERRAT score of both muteins was higher than that of the native BoNT/A. Overall, these validation results indicated that the $3 D$ model of the mutein was proper.

Although the mutated residues were ensured in the nonconserved region, the stability of the protein must still be evaluated. ProtParam provided some tools to analyze protein 

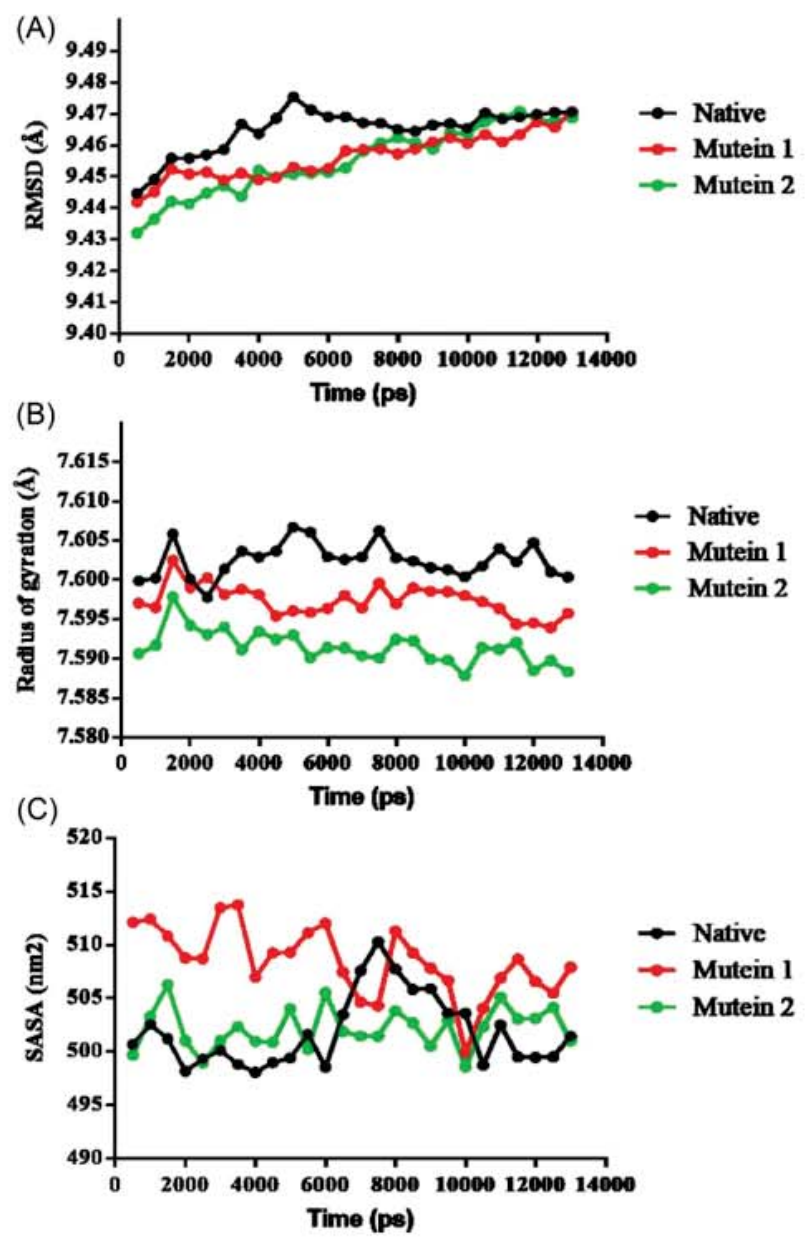

Figure 5. Time evolution of backbone RMSD (A), $R_{g}$ plot (B), and SASA (C) as a function of time for native protein, first-option mutein and second-option mutein.

properties along with the stability (Table 5). Based on the instability index and aliphatic index, both muteins were more stable than the native protein. According to the hydropathy analysis by GRAVY, both muteins had higher scores which signify a more hydrophobic nature than the native, suggesting the lower number of residues on the surface and thus reducing antigenicity (Shi et al., 2015). Instability index led to empiric experiment and algorithm, while aliphatic index was more related to the thermostability of protein due to the hydrophobic stabilization (Ikai, 1980). In this case, the aliphatic index implied that the first-option mutein had better thermostability compared to the other two proteins.

\section{3. $M D$ simulation}

MD analysis is one of the most frequently used methods to determine protein stability in solvated condition. Gromos $53 a 6$ force field was reported to give a better agreement with wet-lab work for Histatin 5 (Henriques, Cragnell, \& Skepö, 2015). Generally, RMSD of the protein backbone can be plotted against time to predict stability. The RMSD plot can be seen in Figure 5(A). A similar trend of the sudden increment of deviation from the beginning of the simulation until the time period of $2.5 \mathrm{~ns}$ also occurred. There was a sharp increase of RMSD for the native BoNT/A in the time period of $5 \mathrm{~ns}$, which reached $\sim 9.475 \AA$. The RMSD values of the native protein reached stability after $6 \mathrm{~ns}$, in which the RMSD values ranged from $\sim 9.465$ to $\sim 9.47 \AA$. Until $10 \mathrm{~ns}$ of simulation time, the RMSD values of both muteins were lower than those of the native one. The RMSD values of the muteins gradually increased in a small difference until the end of the simulation. This small difference in the fluctuations after the relaxation period led to stable trajectories in the simulation. The two muteins showed more deviations from the initial structure. However, the deviation of the native protein was only a maximum of $5 \mathrm{~ns}$. At the end of the RMSD simulation, the RMSD values of the muteins and native protein were similar, though. The values were about $\sim 4.7 \AA$ for both native BoNT/A and the first-option mutein, and $4.68 \AA$ for the second-option mutein.

The $R_{g}$ is the overall size of the protein, computed by measuring all the mass-weight root-mean-square distance from all atoms from the centre of the mass (Rajendran \& Sethumadhavan, 2014). The average $R_{g}$ of both muteins was 

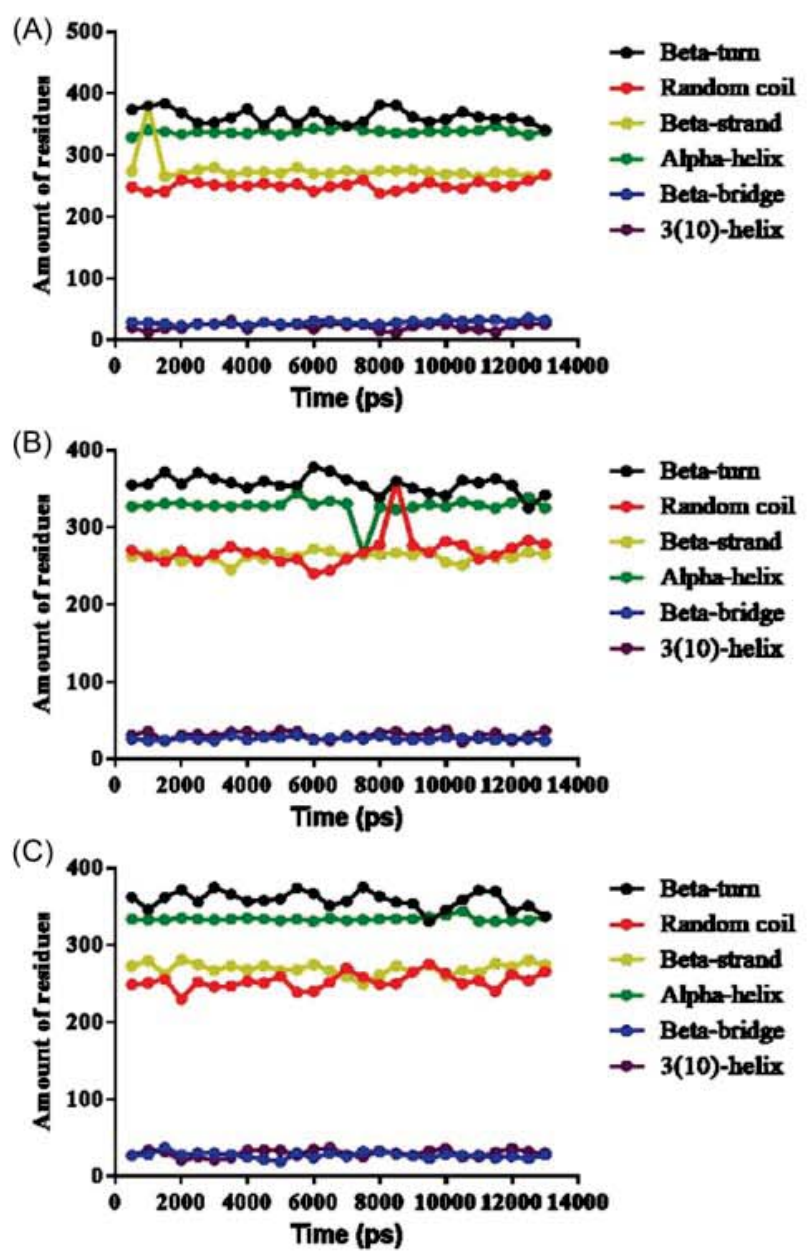

Figure 6. Time evolution of the amount of amino acids residue that contributed to secondary structure of the native BoNT/A (A), mutein first option (B) and mutein second option (C).

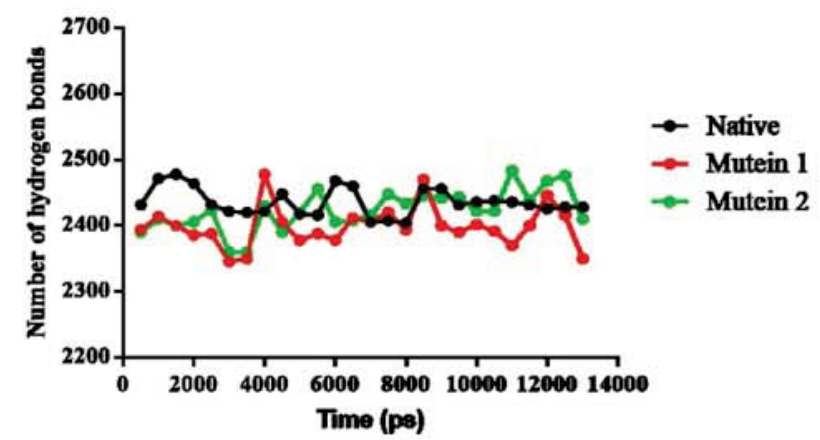

Figure 7. The number of protein intramolecular hydrogen bonds in the native and mutated BoNT/A vs. time at $300 \mathrm{~K}$.

smaller than the native BoNT/A, indicating more compactness of the mutant (Table 6). The range of $R_{g}$ for the native protein was between 7.59783 and $7.6067 \AA$. The range of the first-option mutein was between 7.59394 and $7.60253 \AA$, while the range of the second-option mutein was between 7.58789 and $7.59786 \AA$ (Figure $5(B)$ ). Both muteins were more compact compared to the native protein. By counting the differential of the maximum and minimum $R_{g}$ of all proteins, the mutein in the second option was more flexible than the native, but the mutein in the first option was more rigid than the native one. Nonetheless, all the differences did not even reach $0.01 \AA$. 

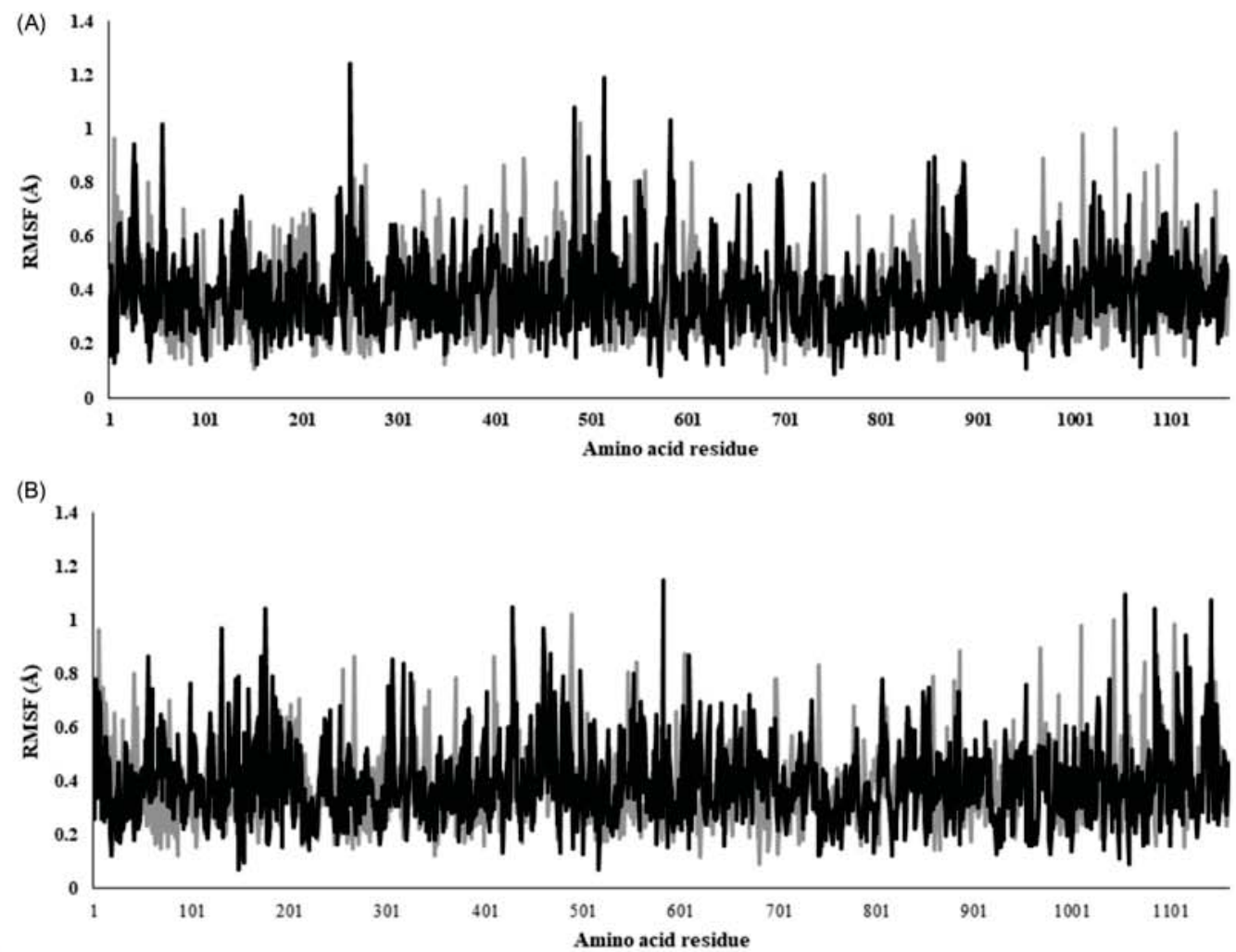

Figure 8. Comparison of the RMSF plot of each residue of (A) native BoNT/A (grey line) vs. first-option mutein (black line) and (B) native BoNT/A (grey line) vs. second-option mutein (black line).

Another parameter to measure the geometry of protein is SASA. The SASA value of native BoNT/A was comparatively similar to the second-option mutein, whereas the SASA value of the first-option mutein was higher than that of the native BoNT/A (Table 6). While the native BoNT/A and the firstoption mutein underwent a change in surface residues in the simulation period, the second-option mutein SASA value was relatively constant, indicating less transition of conformation (Figure $5(C)$ ). This fact was reinforced with the secondary structure prediction along the projection time (Figure 6). The second-option mutein showed less alteration in the secondary structure, implying more conformational stability.

Hydrogen bonds played an important role in assessing the stability of the protein. In the trajectory, the range of the amount of intramolecular hydrogen bonds for native was between 2404 and 2478 bonds, that of the first-option mutein was 2346-2478 bonds, and that of the second-option mutein was between 2360 and 2484 bonds. The fluctuation of intramolecular hydrogen bonds can be seen in Figure 7 , where the native BoNT/A is apt to maintain its amount of hydrogen bonds during the simulation, indicating more rigidity compared to the two muteins.

The flexible regions were located with the help of RMSF of all residues. The RMSF values of native BoNT/A and the muteins were comparable (Figure 8) although both muteins gained more flexibility compared to the native protein (Table 6). Native BoNT/A and both muteins generated similar RMSF mean and deviation of $0.3783 \pm 0.1405 \AA$ for native BoNT/A, $0.3833 \pm 0.1442 \AA$ for the first-option mutein and $0.3892 \pm$ $0.1537 \AA$ for the second-option mutein. Nevertheless, all of the RMSF values were small and below $1 \mathrm{~nm}$, indicating stability of the protein (Nezafat et al., 2016). However, many of the RMSF values were above average, indicating flexibility, and, indeed, BoNT/A was a flexible protein (Silvaggi et al., 2007).

Based on the RMSD, RMSF Rg, SASA, secondary structure prediction and intramolecular hydrogen bond plot, it is evident that the mutation gave more flexibility to the protein in general. The difference was not large; thus, the gain of the flexibility did not disturb the functional behaviors of the protein (Kamaraj \& Purohit, 2013). The mutation did not exhibit destructive impacts to the protein orientation and the function since both BoNT/A mutation options were allowed to make the protein less immunogenic.

\section{Conclusion}

The in silico mutagenesis to generate less immune-reactive BoNT/A has been proposed. There were two proposed options. The first option was $\triangle E 147, \mathrm{E} 510 \mathrm{~F}, \mathrm{~T} 1062 \mathrm{~F}, \Delta E 1080$, $\mathrm{N} 1089 \mathrm{M}$, and $\triangle Q 1090$ which generate the lowest antigenicity score for the overall protein. The second one was $\triangle E 147$, E510F, T1062F, E1080W, N1089M, $\Delta Q 1090$ which was the second lowest antigenicity score for the overall protein. 
However, the antigenicity score for the hotspot residue of 1079-1092 was much lower than that of the first option. Both muteins showed better stability in relation to instability index, aliphatic index and hydropathy analysis compared to the native protein. The MD simulation indicated that the mutation is more flexible to the protein; thus, it would not disrupt the functionality of the protein. These suggestions can be used as rough guidelines for the experimental mutagenesis to save time and cost. In addition, the results of this study provide a breakthrough of better therapeutic and cosmetic suitability through the properties of its BoNT/ A muteins.

\section{Acknowledgements}

We thank Helen Hendaria Kamandhari, Ph.D., for her proofreading and comments.

\section{Disclosure statement}

No potential conflict of interest was reported by the authors.

\section{Author contributions}

Both SEET and YMV wrote the manuscript. Regarding the work collaboration, SEET conducted the mutagenesis, the $3 \mathrm{D}$ modelling and the stability analysis; YMV determined which consensus sequence to be mutated, the occurrence of the mutation (mutagenesis) and the analysis of the immunogenicity of the muteins; and SEDP supervised the work.

\section{Funding}

We would like to thank the Faculty of Biotechnology of Universitas Surabaya for the funding.

\section{References}

Ansari, H. R., \& Raghava, G. P. (2010). Identification of conformational Bcell epitopes in an antigen from its primary sequence. Immunome Research, 6(1), 6-9. doi:10.1186/1745-7580-6-6

Arnold, K., Bordoli, L, Kopp, J. \& Schwede, T. (2006). The SWISS-MODEL workspace: A web-based environment for protein structure homology modelling. Bioinformatics, 22(2), 195-201. doi:10.1093/bioinformatics/ bti770

Babiloni, A. H., Kapos, F. P., \& Nixdorf, D. R. (2016). Intraoral administration of botulinum toxin for trigeminal neuropathic pain. Oral Surgery, Oral Medicine, Oral Pathology and Oral Radiology, 121(6), e148-e153. doi:10.1016/j.0000.2016.03.013

Bazmara, H., Rasooli, I., Jahangiri, A., Sefid, F., Astaneh, S. D. A., \& Payandeh, Z. (2017). Antigenic properties of iron regulated proteins in Acinetobacter baumannii: An in silico approach. International Journal of Peptide Research and Therapeutics, 1-9 [published online]. doi: 10.1007/s10989-017-9665-6

Benkert, P., Biasini, M., \& Schwede, T. (2011). Toward the estimation of the absolute quality of individual protein structure models. Bioinformatics, 27(3), 343-350. doi:10.1093/bioinformatics/btq 662

Benkert, P., Tosatto, S. C., \& Schomburg, D. (2008). QMEAN: A comprehensive scoring function for model quality assessment. Proteins: Structure, Function, and Bioinformatics, 71(1), 261-277. doi:10.1002/ prot.21715

Bentivoglio, A. R., Del Grande, A., Petracca, M., lalongo, T., \& Ricciardi, L. (2015). Clinical differences between botulinum neurotoxin type $A$ and B. Toxicon, 107, 77-84. doi:10.1016/j.toxicon.2015.08.001
Biasini, M., Bienert, S., Waterhouse, A., Arnold, K., Studer, G., Schmidt, T. ... Schwede, T. (2014). SWISS-MODEL: Modelling protein tertiary and quaternary structure using evolutionary information. Nucleic Acids Research, 42(W1), W252-W258. doi:10.1093/nar/gku340

Bowie, J. U., Lüthy, R., \& Eisenberg, D. (1991). A method to identify protein sequences that fold into a known three-dimensional structure. Science, 253(5016), 164-170. doi:10.1126/science.1853201

Brooks, V. B. (1954). The action of botulinum toxin on motor-nerve filaments. The Journal of Physiology, 123(3), 501-515. doi:10.1113/ jphysiol.1954.sp005067

Capriotti, E., Fariselli, P., \& Casadio, R. (2005). I-Mutant2. 0: Predicting stability changes upon mutation from the protein sequence or structure. Nucleic Acids Research, 33(Web Server), W306-W310. doi:10.1093/nar/ gki375

Careta, M. F., Delgado, L., \& Patriota, R. (2015). Report of allergic reaction after application of botulinum toxin. Aesthetic Surgery Journal, 35(5), NP102-NP105. doi:10.1093/asj/sju105

Carruthers, A., \& Carruthers, J. (2005). Botulinum toxin type A. Journal of the American Academy of Dermatology, 53(2), 284-290. doi:10.1016/ j.jaad.2005.03.060

Castelao, M., Marques, R. E., Duarte, G. S., Rodrigues, F. B., Ferreira, J., Sampaio, C., ... Costa, J. (2017). Botulinum toxin type A therapy for cervical dystonia. Cochrane Database of Systematic Reviews, 12 CD003633. doi:10.1002/14651858.CD003633.pub3.

Colovos, C., \& Yeates, T. O. (1993). Verification of protein structures: Patterns of nonbonded atomic interactions. Protein Science, 2(9), 1511-1519. doi:10.1002/pro.5560020916

Doytchinova, I. A., \& Flower, D. R. (2007). Vaxilen: A server for prediction of protective antigens, tumour antigens and subunit vaccines. $B M C$ Bioinformatics, 8(4), 1-7. doi:10.1186/1471-2105-8-4

Dressler, D. (2016). Botulinum toxin drugs: Brief history and outlook Journal of Neural Transmission, 123(3), 277-279. doi:10.1007/500702015-1478-1

Dressler, D., \& Bigalke, H. (2017). Immunological aspects of botulinum toxin therapy. Expert Review of Neurotherapeutics, 17(5), 487-494. doi: $10.1080 / 14737175.2017 .1262258$

Eisenberg, D., Lüthy, R., \& Bowie, J. U. (1997). VERIFY3D: Assessment of protein models with three-dimensional profiles. Methods in Enzymology, 277, 396-404. doi:10.1016/S0076-6879(97)77022-8

Emini, E. A., Hughes, J. V., Perlow, D., \& Boger, J. (1985). Induction of hepatitis $A$ virus-neutralizing antibody by a virus-specific synthetic peptide. Journal of Virology, 55(3), 836-839.

Fattahian, Y., Riahi-Madvar, A., Mirzaee, R., Asadikaram, G., \& Rahbar, M. R. (2017). In silico locating the immune-reactive segments of Lepidium draba peroxidase and designing a less immune-reactive enzyme derivative. Computational Biology and Chemistry, 70, 21-30. doi:10.1016/j.compbiolchem.2017.07.003

Fonfria, E., Elliott, M., Beard, M., Chaddock, J., \& Krupp, J. (2018). Engineering botulinum toxins to improve and expand targeting and SNARE cleavage activity. Toxins, 10(7), 278. doi:10.3390/ toxins 10070278

Frevert, J. (2015). Pharmaceutical, biological, and clinical properties of botulinum neurotoxin type A products. Drugs in R\&D, 15(1), 1-9. doi: 10.1007/s40268-014-0077-1

Gasteiger, E., Hoogland, C., Gattiker, A., Wilkins, M. R., Appel, R. D., \& Bairoch, A. (2005). Protein identification and analysis tools on the ExPASy server. In: Walker, J. M. (Ed.), The proteomics protocols hand book (pp. 571-607). New York, NY: Humana press. doi:10.1385/159259-890-0:571

Hajighahramani, N., Nezafat, N., Eslami, M., Negahdaripour, M., Rahmatabadi, S. S., \& Ghasemi, Y. (2017). Immunoinformatics analysis and in silico designing of a novel multi-epitope peptide vaccine against Staphylococcus aureus. Infection, Genetics and Evolution, 48, 83-94. DOI: 10.1016/j.meegid.2016.12.010 doi:10.1016/ j.meegid.2016.12.010

Henriques, J., Cragnell, C., \& Skepö, M. (2015). Molecular dynamics simulations of intrinsically disordered proteins: Force field evaluation and comparison with experiment. Journal of Chemical Theory and Computation, 117(7), 3420-3431. doi:10.1021/ct501178z 
Hill, K. K., Xie, G., Foley, B. T., \& Smith, T. J. (2015). Genetic diversity within the botulinum neurotoxin-producing bacteria and their neurotoxins. Toxicon, 107, 2-8. doi:10.1016/j.toxicon.2015.09.011

Hospital, A., Andrio, P., Fenollosa, C., Cicin-Sain, D., Orozco, M., \& Gelpí, J. L. (2012). MDWeb and MDMoby: An integrated web-based platform for molecular dynamics simulations. Bioinformatics, 28(9), 1278-1279. doi:10.1093/bioinformatics/bts 139

Ikai, A. (1980). Thermostability and aliphatic index of globular proteins. The Journal of Biochemistry, 88(6), 1895-1898. doi:10.1093/ oxfordjournals.jbchem.a133168

Jespersen, M. C., Peters, B., Nielsen, M., \& Marcatili, P. (2017). BepiPred2.0: Improving sequence-based B-cell epitope prediction using conformational epitopes. Nucleic Acids Research, 45(W1), W24-W29. doi: $10.1093 / \mathrm{nar} / \mathrm{gkx} 346$

Kalyanaraman, N. (2018). In silico prediction of potential vaccine candidates on capsid protein of human bocavirus 1. Molecular Immunology, 93, 193-205. doi:10.1016/j.molimm.2017.11.024

Kamaraj, B., \& Purohit, R. (2013). In silico screening and molecular dynamics simulation of disease-associated nsSNP in TYRP1 gene and its structural consequences in OCA3. BioMed Research International, 2013, 1 (article ID 697051). doi:10.1155/2013/697051

Kelley, L. A., Mezulis, S., Yates, C. M., Wass, M. N., \& Sternberg, M. J. (2015). The Phyre2 web portal for protein modeling, prediction and analysis. Nature Protocols, 10(6), 845-858. doi:10.1038/nprot.2015.053

Kolaskar, A. S., \& Tongaonkar, P. C. (1990). A semi-empirical method for prediction of antigenic determinants on protein antigens. FEBS Letters, 276(1-2), 172-174. doi:10.1016/0014-5793(90)80535-0

Kringelum, J. V., Lundegaard, C., Lund, O., \& Nielsen, M. (2012). Reliable B cell epitope predictions: Impacts of method development and improved benchmarking. PLoS Computational Biology, 8(12), e1002829. doi:10.1371/journal.pcbi.1002829

Lacy, D. B., Tepp, W., Cohen, A. C., DasGupta, B. R., \& Stevens, R. C. (1998). Crystal structure of botulinum neurotoxin type A and implications for toxicity. Nature Structural Biology, 5(10), 898-902. DOl: $10.1038 / 2338$ doi: $10.1038 / 2338$

Larsen, J. E. P., Lund, O., \& Nielsen, M. (2006). Improved method for predicting linear B-cell epitopes. Immunome Research, 2(1), 2-7. doi: 10.1186/1745-7580-2-2

Lewitt, P. A., \& Trosch, R. M. (1997). Idiosyncratic adverse reactions to intramuscular botulinum toxin type A injection. Movement Disorders, 12(6), 1064-1067. doi:10.1002/mds.870120637

Li, M., Goldberger, B. A., \& Hopkins, C. (2005). Fatal case of BOTOX related anaphylaxis? Journal of Forensic Sciences, 50(1), 169-172. doi: $10.1520 / J F S 2004196$

Lüthy, R., Bowie, J. U., \& Eisenberg, D. (1992). Assessment of protein models with three-dimensional profiles. Nature, 356(6364), 83. doi: $10.1038 / 356083 a 0$

Luvisetto, S., Gazerani, P., Cianchetti, C., \& Pavone, F. (2015). Botulinum toxin type $A$ as a therapeutic agent against headache and related disorders. Toxins, 7(9), 3818-3844. doi:10.3390/toxins7093818

Moon, I. J., Chang, S. E., \& Kim, S. D. (2017). First case of anaphylaxis after botulinum toxin type $\mathrm{A}$ injection. Clinical and Experimental Dermatology, 42(7), 760-762. doi:10.1111/ced.13108

Negahdaripour, M., Eslami, M., Nezafat, N., Hajighahramani, N., Ghoshoon, M. B., Shoolian, E., ... Ghasemi, Y. (2017). A novel HPV prophylactic peptide vaccine, designed by immunoinformatics and structural vaccinology approaches. Infection, Genetics and Evolution, 54, 402-416. doi:10.1016/j.meegid.2017.08.002

Nezafat, N., Karimi, Z., Eslami, M., Mohkam, M., Zandian, S., \& Ghasemi, Y. (2016). Designing an efficient multi-epitope peptide vaccine against Vibrio cholerae via combined immunoinformatics and protein interaction-based approaches. Computational Biology and Chemistry, 62, 82-95. doi:10.1016/j.compbiolchem.2016.04.006

Pahil, S., Taneja, N., Ansari, H. R., \& Raghava, G. P. S. (2017). In silico analysis to identify vaccine candidates common to multiple serotypes of Shigella and evaluation of their immunogenicity. PloS One, 12(8), e0180505. doi:10.1371/joumal.pone.0180505
Potocnakova, L., Bhide, M., \& Pulzova, L. B. (2016). An introduction to Bcell epitope mapping and in silico epitope prediction. Journal of Immunology Research, 2016(6760830), 1-11. doi:10.1155/2016/6760830 Rajendran, V., \& Sethumadhavan, R. (2014). Drug resistance mechanism of PncA in Mycobacterium tuberculosis. Journal of Biomolecular Structure and Dynamics, 32(2), 209-221. doi:10.1080/ 07391102.2012 .759885

Rigoni, M., Caccin, P., Johnson, E. A., Montecucco, C., \& Rossetto, O. (2001). Site-directed mutagenesis identifies active-site residues of the light chain of botulinum neurotoxin type A. Biochemical and Biophysical Research Communications, 288(5), 1231-1237. doi:10.1006/ bbrc.2001.5911

Rosenfield, L. K., Kardassakis, D. G., Tsia, K. A., \& Stayner, G. (2014). The first case report of a systemic allergy to OnabotulinumtoxinA (Botox) in a healthy patient. Aesthetic Surgery Journal, 34(5), 766-768. doi: $10.1177 / 1090820 \times 14532648$

Saha, S., \& Raghava, G. P. S. (2006). Prediction of continuous B-cell epito pes in an antigen using recurrent neural network. Proteins: Structure, Function, and Bioinformatics, 65(1), 40-48. doi:10.1002/prot.21078

Schellekens, H. (2002). Immunogenicity of therapeutic proteins: Clinica implications and future prospects. Clinical Therapeutics, 24(11), 1720-1740. doi:10.1016/S0149-2918(02)80075-3

Scott, A. B. (1981). Botulinum toxin injection of eye muscles to correct strabismus. Transactions of the American Ophthalmological Society, 79, 734-770.

Sefid, F., Rasooli, l., Jahangiri, A., \& Bazmara, H. (2015). Functional exposed amino acids of BauA as potential immunogen against Acinetobacter baumannii. Acta Biotheoretica, 63(2), 129-149. doi 10.1007/s10441-015-9251-2

Shi, J., Zhang, J., Li, S., Sun, J., Teng, Y., Wu, M., ... Hu, Y. (2015). Epitope-based vaccine target screening against highly pathogenic MERS-CoV: An in silico approach applied to emerging infectious diseases. PloS One, 10(12), e0144475, doi:10.1371/journal.pone.0144475

Silvaggi, N. R., Boldt, G. E., Hixon, M. S., Kennedy, J. P., Tzipori, S., Janda, K. D., \& Allen, K. N. (2007). Structures of Clostridium botulinum neurotoxin serotype A light chain complexed with small-molecule inhibitors highlight active-site flexibility. Chemistry \& Biology, 14(5), 533-542. doi:10.1016/j.chembiol.2007.03.014

Singh, H., Ansari, H. R., \& Raghava, G. P. (2013). Improved method for lin ear B-cell epitope prediction using antigen's primary sequence. PloS One, 8(5), e62216. doi:10.1371/joumal.pone.0062216

Swearingen, K. E., Lindner, S. E., Shi, L., Shears, M. J., Harupa, A., Hopp, C. S., ... Sinnis, P. (2016). Interrogating the Plasmodium sporozoite surface: Identification of surface-exposed proteins and demonstration of glycosylation on CSP and TRAP by mass spectrometry-based proteomics. PLoS Pathogens, 12(4), e1005606. doi:10.1371/ journal.ppat. 1005606

Wiederstein, M., \& Sippl, M. J. (2007). ProSA-web: Interactive web service for the recognition of errors in three-dimensional structures of proteins. Nucleic Acids Research, 35(Web Server), W407-W410. doi: $10.1093 / \mathrm{nar} / \mathrm{gkm} 290$

Yao, B., Zhang, L., Liang, S., \& Zhang, C. (2012). SVMTriP: A method to predict antigenic epitopes using support vector machine to integrate tri-peptide similarity and propensity. PloS One, 7(9), e45152. doi: 10.1371/journal.pone.0045152

Yasmin, T., Akter, S., Debnath, M., Ebihara, A., Nakagawa, T., \& Nabi, A. N. (2016). In silico proposition to predict cluster of B-and T-cell epitopes for the usefulness of vaccine design from invasive, virulent and membrane associated proteins of C. jejuni. In Silico Pharmacology, 4(5), 1-10. doi:10.1186/540203-016-0020-y

Zarei, M., Nezafat, N., Rahbar, M. R., Negahdaripour, M., Sabetian, S. Morowvat, M. H., \& Ghasemi, Y. (2018). Decreasing the immunogenicity of arginine deiminase enzyme via structure-based computational analysis. Journal of Biomolecular Structure and Dynamics, 1-14 [published online]. doi:10.1080/07391 102.2018.1431151 
in_silico_mutagenesis.pdf

ORIGINALITY REPORT

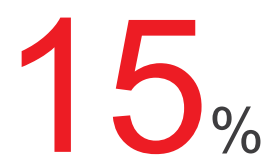

SIMILARITY INDEX
$10 \%$

INTERNET SOURCES
$14 \%$

PUBLICATIONS
$5 \%$

STUDENT PAPERS

MATCH ALL SOURCES (ONLY SELECTED SOURCE PRINTED)

$1 \%$

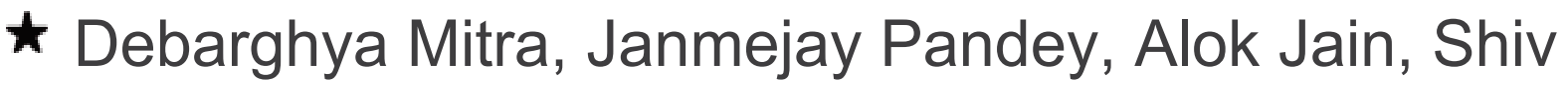
Swaroop. " design of multi-epitope-based peptide vaccine against SARS-CoV-2 using its spike protein ", Journal of Biomolecular Structure and Dynamics, 2021 Publication

Exclude quotes

Exclude bibliography 